\title{
Dark matter to baryon ratio from scalar triplets decay in type-II seesaw
}

\author{
Nimmala Narendra ${ }^{1, a}$, Narendra Sahu ${ }^{2, b}$, Sujay Shil ${ }^{3,4, c}$ \\ ${ }^{1}$ Theoretical Physics Division, Physical Research Laboratory, Ahmedabad 380009, India \\ 2 Department of Physics, Indian Institute of Technology Hyderabad, Kandi, Sangareddy, Telangana 502285, India \\ ${ }^{3}$ Institute of Physics, Sachivalaya Marg, Bhubaneswar, Odisha 751005, India \\ ${ }^{4}$ Homi Bhabha National Institute, Training School Complex, Anushakti Nagar, Mumbai 400085, India
}

Received: 28 June 2021 / Accepted: 27 November 2021 / Published online: 13 December 2021

(C) The Author(s) 2021

\begin{abstract}
We propose a minimal model for the cosmic coincidence problem $\Omega_{\mathrm{DM}} / \Omega_{B} \sim 5$ and neutrino mass in a typeII seesaw scenario. We extend the standard model of particle physics with a SU(2) singlet leptonic Dirac fermion $\chi$, which represents the candidate of dark matter (DM), and two triplet scalars $\Delta_{1,2}$ with hierarchical masses. In the early Universe, the $\mathrm{CP}$ violating out-of-equilibrium decay of lightest $\Delta$ generates a net $B-L$ asymmetry in the visible sector (comprising of SM fields), where $B$ and $L$ represents the total baryon and lepton number respectively. A part of this asymmetry gets transferred to the dark sector (comprising of DM $\chi$ ) through a dimension eight operator which conserves $B-L$. Above the electroweak phase transition, the $B-L$ asymmetry of the visible sector gets converted to a net $B$-asymmetry by the $B+L$ violating sphalerons, while the $B-L$ asymmetry of the dark sector remains untouched which we see today as relics of DM. We show that the observed DM abundance can be explained for a DM mass about $8 \mathrm{GeV}$. We then introduce an additional singlet scalar field $\phi$ which mixes with the SM-Higgs to annihilate the symmetric component of the DM resonantly which requires the singlet scalar mass to be twice the DM mass, i.e. around $16 \mathrm{GeV}$, which can be searched at collider experiments. In our model, the active neutrinos also get small masses by the induced vacuum expectation value (vev) of the triplet scalars $\Delta_{1,2}$. In the later part of the paper we discuss all the constraints on model parameters coming from invisible Higgs decay, Higgs signal strength, DM direct detection and relic density of DM.
\end{abstract}

\footnotetext{
${ }^{a}$ e-mail: nimmalanarendra@gmail.com

b e-mail: nsahu@phy.iith.ac.in (corresponding author)

c e-mail: sujayshil1@gmail.com
}

\section{Introduction}

One of the most important aspects of beyond the standard model (SM) of particle physics is dark matter (DM) phenomenology. There are lots of astrophysical evidences which ensure the existence of DM [1,2]. The prime among them are the galaxy rotation curve, gravitational lensing and the large scale structure of the Universe. Another important puzzle in physics is why the Universe is baryon asymmetric. The baryon asymmetry of the Universe is usually given in terms of $\eta=\frac{n_{B}-n_{\bar{B}}}{n_{\gamma}}=(6.12 \pm 0.04) \times 10^{-10}$ [3], where $n_{\gamma}$ is the photon number density in a comoving volume and is related to entropy density $s$ as $s=7.04 n_{\gamma}$. Here $n_{B}$ and $n_{\bar{B}}$ are respectively baryon and anti baryon densities. The baryon asymmetry of the Universe is maximal today, i.e., $n_{\bar{B}}=0$. As a result $\eta$ can be expressed in terms of baryon abundance $Y_{B}$ as $\eta=7.04 Y_{B}$, where $Y_{B} \equiv n_{B} / s$. The $\mathrm{CP}$ violation within the SM is not adequate to explain the present baryon asymmetry of the Universe. This is another reason why to explore the physics beyond the SM (BSM).

Experimentally it has been observed that the relic density of DM, given in terms of $\Omega_{\mathrm{DM}} \equiv \rho_{\mathrm{DM}} / \rho_{c}$, is about five times larger than the relic density of baryons in the present Universe, i.e., $\Omega_{\mathrm{DM}} \sim 5 \Omega_{\mathrm{B}}$, where $\Omega_{\mathrm{DM}} h^{2}=0.120 \pm 0.001$ and $\Omega_{\mathrm{B}} h^{2}=0.0224 \pm 0.0001[4,5]$. This proportionality is known to be cosmic coincidence problem. The observed DM abundance in a comoving volume can be given as

$$
Y_{\mathrm{DM}} \equiv \frac{n_{\mathrm{DM}}}{s}=4 \times 10^{-10}\left(\frac{1 \mathrm{GeV}}{M_{\mathrm{DM}}}\right)\left(\frac{\Omega_{\mathrm{DM}} h^{2}}{0.11}\right) .
$$

Thus the ratio of the abundances of DM to baryons, i.e., $Y_{\mathrm{DM}} / Y_{B} \approx \mathcal{O}(1)$ for $M_{\mathrm{DM}} \sim 5 \mathrm{GeV}$. However, the $\mathrm{DM}$ mass can vary from a $\mathrm{GeV}$ to a $\mathrm{TeV}$ scale depending on the amount of $\mathrm{CP}$ violation in visible and dark sectors. See 


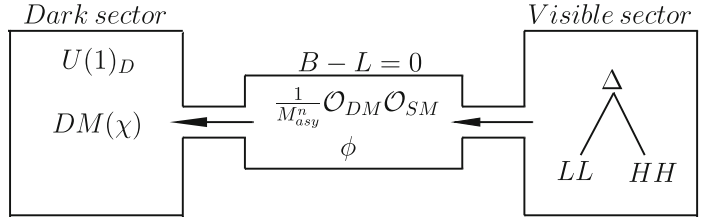

Fig. 1 Pictorial presentation of thermal contact between the dark sector and visible sector via scalar portal as well as higher dimension operator, which conserves $B-L$ symmetry and are in thermal equilibrium above sphaleron decoupling temperature

for instance [6-8]. ${ }^{1}$ The idea of asymmetric DM is similar to the baryon asymmetry of the present Universe, i.e., an asymmetry in DM particle over its anti-particle [6-80] (Fig. $1)$.

In this article we propose a model to explain the cosmic coincidence problem: $\Omega_{\mathrm{DM}} / \Omega_{B} \sim 5$ in a simple extension of the SM. We introduce two SU(2) triplet scalar fields $\left(\Delta_{1,2}\right)$, which decay to the SM lepton doublet and Higgs field [68,81-85] in the early Universe as pictorially shown in Fig. 7. The triplets decay satisfy all the Sakharov conditions [86-89] to give the lepton asymmetry in the SM sector [90]. An extra scalar $\phi$ and a leptonic Dirac fermion $\chi$ have been introduced in the model. The leptonic Dirac fermion $\chi$ is charged under a global $\mathrm{U}(1)_{\mathrm{D}}$ symmetry which provides stability to the DM and also forbids the Majorana mass term of the $\chi$, the term which can spoil the asymmetry in the dark sector. The asymmetry created in the leptonic sector transfers to the DM sector through a dimension-8 operator $\mathcal{O}_{8}=\frac{\bar{\chi}^{2}(L H)^{2}}{M_{a s y}^{4}}[91-$ 94], which conserves $B-L$ symmetry. Since the operator $\mathcal{O}_{8}$ conserves $B-L$, it redistributes the $B-L$ asymmetry, produced by $\Delta$-decay, between SM leptons (visible sector) and $\chi$ (dark sector). Above the electroweak phase transitions, the $B+L$ violating sphaleron processes convert the $B-L$ asymmetry of the visible sector to a net baryon (B) asymmetry that we observe today, while the $B-L$ asymmetry of the dark sector remains untouched which we see today as DM abundance. We show that correct relic density of DM requires its mass to be around $8 \mathrm{GeV}$. The symmetric component of $\chi$ gets annihilated resonantly via $\phi-H$ mixing, which in turns requires the singlet scalar $\phi$ mass to be around twice the DM mass. Note that the dimension eight operator $\mathcal{O}_{8}$ softly breaks the $U(1)_{D}$ global symmetry to a remnant $Z_{2}$ symmetry under which $\chi$ is odd and all other particles are even. In this way, we ensure the stability to DM as well as the theory escapes from having a Goldstone boson.

The advantages of considering an asymmetric DM in this paper are as follows: (1) The hitherto null detection of WIMP

\footnotetext{
${ }^{1}$ In Refs. [6-8] an inert fermion doublet $\psi$ was introduced which symmetrically couples to $\Delta$. If $\psi$ is odd under a remnant $Z_{2}$ discrete symmetry, then the neutral component of $\psi$ can be a candidate of inelastic asymmetric DM.
}

DM (with a typical mass $\mathcal{O}(100) \mathrm{GeV}$ and having a weak interaction cross-section) at leading direct DM search experiments like XENON1T [95] attracts many other experiments probing DM at low mass regime, where XENON1T is insensitive. The prime among them are CRESST-II [96], EDELWEISS [97], CDMS-II [98], CoGeNT [99], DAMIC [100], SuperCDMS low mass [101], CDMSlite with Ge [102], PandaX-II [103], ZEPLIN-III [104], DarkSide-50 [105] etc. These experiments will shed light on a low mass DM, typically mass less than $10 \mathrm{GeV}$ as we have considered in this paper. (2) The other advantage is the search of a light scalar is under active consideration at LHC [106-111]. Therefore, there exist a fair chance that future data can shed light to our model. Since the DM and singlet scalar masses are correlated in our case, the model thus in principle can be probed in future.

The paper is organized as follows: in Sect. 2, we introduce the model part. Section 3 is devoted to explain the neutrino masses in a type-II seesaw framework. We discuss the generation of lepton asymmetry in the visible sector and its transfer to a dark sector in Sect. 4. In Sect. 4.1, we show the condition for depletion of symmetric component of the DM. The constraints from invisible Higgs decay, Higgs signal strength, the relic abundance of DM and its direct detection on the model parameters are discussed in Sects. 4.2, 4.3, 4.4, respectively. In Sect. 6 we conclude.

\section{The model}

Here we extend the SM of particle physics with a $U(1)_{D}$ global symmetry. The additional particle content that we introduce to the SM are: two triplet scalars $\Delta_{1,2}$, a singlet leptonic Dirac fermion $\chi$ and a $S U(2)_{L}$ singlet scalar $\phi$. The latter mixes with the SM-Higgs $H$ and provides interesting low energy phenomenology as we discuss in following sections. The $U(1)_{D}$ global symmetry is softly broken by the dimension eight operator ${ }^{2} \mathcal{O}_{8}=\frac{\overline{\bar{\chi}}^{2}(L H)^{2}}{M_{a s y}^{4}}$ to a remnant $Z_{2}$ symmetry under which the leptonic Dirac fermion $\chi$ is odd while all other particles are even. As a result $\chi$ is stable and represents a candidate of DM. The details of dimension eight operator $\mathcal{O}_{8}$ will be discussed in Sect. 4. The particle content of the model and the corresponding quantum numbers are given in the Table 1.

The Lagrangian of our model can be written as:

$$
\begin{aligned}
& \mathcal{L} \supset \bar{\chi} i \gamma^{\mu} \partial_{\mu} \chi+\left(\partial_{\mu} \phi\right)\left(\partial^{\mu} \phi\right)-M_{\chi} \bar{\chi} \chi-\lambda_{\mathrm{DM}} \bar{\chi} \chi \phi \\
& -\lambda \overline{\left(L^{c}\right)} i \tau^{2} \Delta L-V(H, \phi),
\end{aligned}
$$

\footnotetext{
2 We have given a viable origin of dimension- 8 operator in the Appendix B.
} 
Table 1 Quantum numbers of the new particles under the imposed symmetry

\begin{tabular}{lllll}
\hline Fields & $\mathrm{SU}(3)_{c}$ & $\mathrm{SU}(2)_{L}$ & $\mathrm{U}(1)_{Y}$ & $U(1)_{D}$ \\
\hline$\Delta$ & 1 & 3 & +1 & 0 \\
$\phi$ & 1 & 1 & 0 & 0 \\
$\chi$ & 1 & 1 & 0 & -1 \\
\hline
\end{tabular}

where

$$
\begin{aligned}
V(H, \phi)= & -\mu_{H}^{2} H^{\dagger} H+\lambda_{H}\left(H^{\dagger} H\right)^{2}+M_{\phi}^{2} \phi^{2} \\
& +\lambda_{\phi} \phi^{4}+M_{\Delta}^{2} \Delta^{\dagger} \Delta+\lambda_{\Delta}\left(\Delta^{\dagger} \Delta\right)^{2} \\
& +\left[\mu \overline{\left(H^{c}\right)} i \tau^{2} \Delta^{\dagger} H+h . c .\right] \\
& +\rho_{1} \phi\left(H^{\dagger} H\right)+\lambda_{H \phi}\left(H^{\dagger} H\right) \phi^{2} \\
& +\rho_{2} \phi\left(\Delta^{\dagger} \Delta\right)+\lambda_{H \Delta}\left(H^{\dagger} H\right)\left(\Delta^{\dagger} \Delta\right) \\
& +\lambda_{\Delta \phi}\left(\Delta^{\dagger} \Delta\right) \phi^{2} .
\end{aligned}
$$

Here we assume the mass of $\Delta$ to be super heavy as we need to explain the small neutrino masses (see Sect. 3) [82]. Therefore $\Delta$ does not play any role in the low energy phenomenology. The necessary and sufficient conditions for the vacuum stability of the potential are $[112,113]$ :

$$
\begin{aligned}
& \lambda_{H} \geq 0, \quad \lambda_{\phi} \geq 0, \quad \lambda_{\Delta} \geq 0, \\
& \tilde{\lambda_{1}}=\frac{1}{2} \lambda_{H \Delta}+\sqrt{\lambda_{H} \lambda_{\Delta}} \geq 0, \\
& \tilde{\lambda_{2}}=\frac{1}{2} \lambda_{H \phi}+\sqrt{\lambda_{H} \lambda_{\phi}} \geq 0, \\
& \tilde{\lambda_{3}}=\frac{1}{2} \lambda_{\Delta \phi}+\sqrt{\lambda_{\Delta} \lambda_{\phi}} \geq 0, \\
& \sqrt{\lambda_{H} \lambda_{\Delta} \lambda_{\phi}}+\frac{1}{2} \lambda_{H \Delta} \sqrt{\lambda_{\phi}} \\
& +\frac{1}{2} \lambda_{H \phi} \sqrt{\lambda_{\Delta}}+\frac{1}{2} \lambda_{\Delta \phi} \sqrt{\lambda_{H}}+\sqrt{2 \tilde{\lambda_{1}} \tilde{\lambda_{2}} \tilde{\lambda_{3}}} \geq 0 .
\end{aligned}
$$

We assume $M_{\phi}^{2}$ to be positive, so that $\phi$ does not acquire any direct vev. However, the electroweak phase transition induces a non-zero vev to $\phi$ due to the trilinear term $\rho_{1} \phi\left(H^{\dagger} H\right)$ as given in Eq. 3. We assume that $\langle\phi\rangle=u<<v$, where $v$ is the SM-Higgs vev. The quantum fluctuation of these fields around the minimum can be given as:

$H=\left(\begin{array}{c}0 \\ \frac{v+h}{\sqrt{2}}\end{array}\right)$ and $\phi=\frac{u+\tilde{\phi}}{\sqrt{2}}$.

Minimizing the scalar potential 3, we get the vevs

$v=\sqrt{\frac{\mu_{H}^{2}-\frac{1}{2} \lambda_{H \phi} u^{2}-\frac{1}{\sqrt{2}} \rho_{1} u}{\lambda_{H}}}$, and

$u=-\frac{\rho_{1} v^{2}}{2 \sqrt{2}\left(M_{\phi}^{2}+\frac{1}{2} \lambda_{H \phi} v^{2}\right)}$.

Thus from the above equation we see that as $\rho_{1} \rightarrow 0$, the induced vev $u \rightarrow 0$. We note that the non zero vev of $\phi$ does not affect the discussion in the following sections, ${ }^{3}$ hence we set it to be zero (i.e., $\langle\phi\rangle=0$ ) for simplicity. As a result Eq. 9 can be rewritten as:

$H=\left(\begin{array}{c}0 \\ \frac{v+h}{\sqrt{2}}\end{array}\right)$ and $\phi=\tilde{\phi} / \sqrt{2}$.

Due to $\tilde{\phi}-h$ mixing we get the mass matrix:

$\left(\begin{array}{cc}2 \lambda_{H} v^{2} & \frac{\rho_{1} v}{\sqrt{2}} \\ \frac{\rho_{1} v}{\sqrt{2}} & M_{\phi}^{2}+\frac{\lambda_{H \phi}}{2} v^{2}\end{array}\right)$.

After diagonalising the mass matrix, given by Eq. 13, we get the two mass eigenstates:

$h_{1}=h \cos \gamma+\tilde{\phi} \sin \gamma$

$h_{2}=-h \sin \gamma+\tilde{\phi} \cos \gamma$.

We identify $h_{1}$ to be the SM-like Higgs with mass $M_{h_{1}}=$ $125.18 \mathrm{GeV}$, while $h_{2}$ remains as the second Higgs whose mass is going to be determined from the required phenomenology. In particular, we determine $h_{2}$ mass from relic abundance requirement. In Sect. 4.1 we obtain it's mass as per the requirement of depletion of the symmetric component of the DM to be $M_{h_{2}} \approx 2 M_{\chi} \approx 16 \mathrm{GeV}$. The $\tilde{\phi}-h$ mixing obtained from Eq. 13 is given by:

$\sin \gamma \approx \frac{\rho_{1} v / \sqrt{2}}{2 \lambda_{H} v^{2}-M_{\phi}^{2}-\frac{\lambda_{H \phi} v^{2}}{2}}$.

From Eqs. 13 and 15 we see that $M_{h_{1}}, M_{h_{2}}$ and $\sin \gamma$ primarily depend on $\rho_{1}, \lambda_{H}, \lambda_{H \phi}$. Without loss of generality we set $M_{\phi}=0$. In the right panel of Fig. 2 we show the contours of $M_{h_{1}}=125 \mathrm{GeV}$ (dashed lines) and $M_{h_{2}}=16.425 \mathrm{GeV}$ (solid lines) and $\sin \gamma=0.16,0.6$ (dot-dashed lines) in the plane of $\lambda_{H}$ versus $\rho_{1}$ for $\lambda_{H \phi}=0.01$ (meeting at point B) and 0.1 (meeting at point $\mathrm{A}$ ). Thus we see that a large range of $\sin \gamma$ is allowed to explain simultaneously the masses of $h_{1}$ and $h_{2}$. However, we shall show in Sects. 4.2-4.4 that $\sin \gamma \gtrsim 0.2$ is not allowed.

\footnotetext{
${ }^{3}$ Non-zero vev of $\phi$ only modifies the mass of the dark matter which ultimately fixed by cosmic co-incidence condition but all the other discussions will be the same. Moreover, the non-zero vev of $\phi$ does not affect any low energy phenomenological calculations such as dark matter annihilation and direct detection, bound from the Higgs measurement etc. Therefore, we set the vev of $\phi$ to be zero for simplicity.
} 
Fig. 2 Left: $\lambda_{e f f}(\mathrm{GeV})$ as a function of $\sin \gamma$. Right: Simultaneous solution to the masses of $h_{1}$ and $h_{2}$ for different set of values of $\sin \gamma$ and $\lambda_{H \phi}$
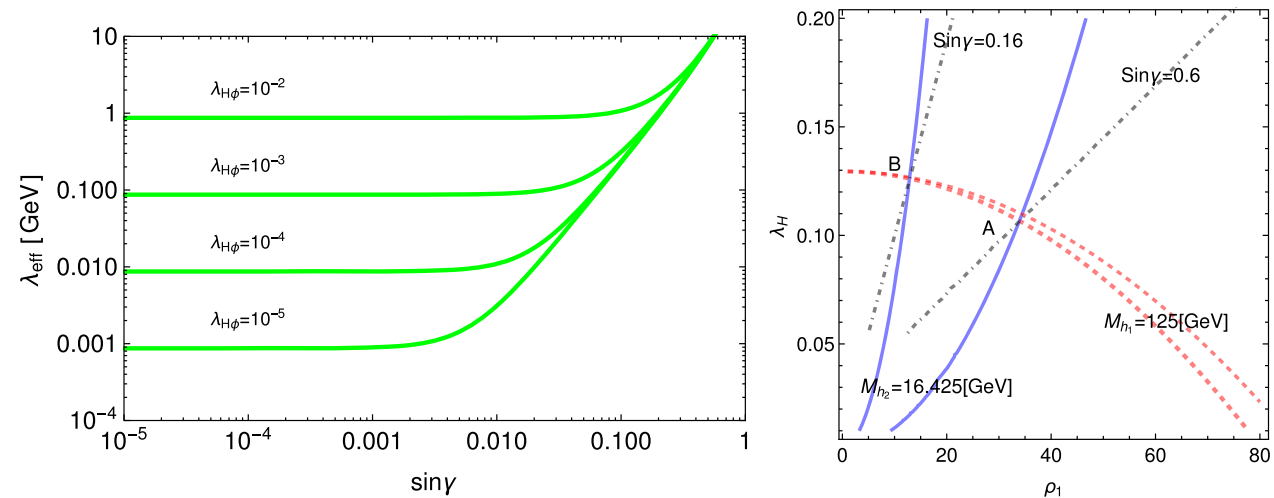

Now we write the effective coupling of $h_{1} h_{2} h_{2}$-vertex which is relevant for the collider signature of $h_{2}$, studied in Sect. 5. Form Eq. 3, the $h_{1} h_{2} h_{2}$-vertex can be derived as:

$$
\begin{aligned}
\lambda_{\text {eff }}= & 3 \lambda_{H} v \cos \gamma \sin ^{2} \gamma+\frac{\lambda_{H \phi}}{2} v \cos ^{3} \gamma+\frac{\rho_{1}}{2 \sqrt{2}} \sin ^{3} \gamma \\
& -\frac{\rho_{1}}{\sqrt{2}} \sin \gamma \cos ^{2} \gamma-\lambda_{H \phi} v \sin ^{2} \gamma \cos \gamma .
\end{aligned}
$$

In the left-panel of Fig. 2 we show $\lambda_{\text {eff }}$ as a function of $\sin \gamma$ for different values of $\lambda_{H \phi}$. One can see that $\lambda_{\text {eff }}$ is almost independent of $\lambda_{H \phi}$ for $\sin \gamma \gtrsim 0.1$. We will use it to obtain the production cross-section of $h_{2}$ in Sect.5.

\section{Neutrino masses}

This model also explains the sub-eV neutrino masses of light neutrinos through the type-II seesaw [114-119]. The relevant terms in the Lagrangian (2) are given as:

$\mathcal{L} \supset M_{\Delta}^{2} \Delta^{\dagger} \Delta+\lambda \overline{\left(L^{c}\right)} i \tau^{2} \Delta L+\mu \overline{\left(H^{c}\right)} i \tau^{2} \Delta^{\dagger} H+$ h.c..

Since we assume $M_{\Delta}^{2}>0$, so $\Delta$ does not acquire any direct vev. However, after electroweak phase transition, the trilinear term $\mu \Delta^{\dagger} H H$ induces a non-zero vev to $\Delta$ as:

$\langle\Delta\rangle \equiv w \simeq \frac{-\mu v^{2}}{M_{\Delta}^{2}}$,

Where $v=\langle H\rangle=246 \mathrm{GeV}$. This can be verified by minimising the scalar potential, Eq. 3. Note that the sign of $\langle\Delta\rangle$ in Eq. 18 is not important ${ }^{4}$ as it gives an overall phase, $e^{i \pi}$ (which is not a physical quantity), to the neutrino mass through the relation

$\left(M_{\nu}\right)_{\alpha \beta}=\lambda_{\alpha \beta}\langle\Delta\rangle=\left(\lambda_{\alpha \beta} \frac{-\mu v^{2}}{M_{\Delta}^{2}}\right)$.

\footnotetext{
${ }^{4}$ See for example: [81-85] for negative sign of $\langle\Delta\rangle$ and $[119,120]$ for the positive sign of $\langle\Delta\rangle$.
}

For $\lambda \approx \mathcal{O}(1)$, to get observed light neutrino masses, we choose $\mu \sim M_{\Delta} \sim 10^{14} \mathrm{GeV}$. Note that the electroweak $\rho$ parameter constrains the vev of $\Delta$ to satisfy the requirement of

$\rho \equiv \frac{M_{w}^{2}}{M_{Z}^{2} \cos \theta_{w}}=\frac{1+2 x^{2}}{1+4 x^{2}} \approx 1$

where $x=w / v$. This implies, $w<\mathcal{O}(1) \mathrm{GeV}$.

\section{Triplet scalar leptogenesis and asymmetric DM}

In the early Universe the triplet scalars are assumed to be in thermal equilibrium at a temperature above their mass scales. As the Universe expands and the temperature falls below the mass scale of a triplet scalar, the latter goes out of thermal equilibrium and decays through the processes: $\Delta \rightarrow L L$ and $\Delta \rightarrow H H$. These decay channels combinely violate $B-L$ by two units and hence lead to leptogenesis as we discuss below. The decay rate of $\Delta$ is given by

$\Gamma_{\Delta}=\frac{1}{8 \pi}\left(|\lambda|^{2}+\frac{\mu^{2}}{M_{\Delta}^{2}}\right) M_{\Delta}$

The out of equilibrium condition of $\Delta$ is set by comparing $\Gamma_{\Delta}$ with the Hubble expansion parameter $H=1.67 g_{*}^{1 / 2} T^{2} / M_{\mathrm{Pl}}$ at $T \sim M_{\Delta}$, and it is given by

$|\lambda| \lesssim\left|\sqrt{1.67 \times 8 \pi \sqrt{g_{*}}\left(M_{\Delta} / M_{\mathrm{Pl}}\right)-\left(\mu^{2} / M_{\Delta}^{2}\right)}\right|$.

Here the $g_{*}$ and $M_{\mathrm{Pl}}$ are the total degrees of freedom and the Planck mass respectively. For $\mu \leq M_{\Delta} \simeq 10^{14} \mathrm{GeV}$, we get $|\lambda| \lesssim \mathcal{O}(1)$. The decoupling epoch can be different for different mass scale of triplet scalars.

To get the CP asymmetry we need at least two copies of triplet scalars. In presence of these triplet scalars interactions, the diagonal mass $M_{\Delta}^{2}$ in Eq. 3 can be replaced by [82],

$\frac{1}{2} \Delta_{a}^{\dagger}\left(M_{+}^{2}\right)_{a b} \Delta_{b}+\frac{1}{2}\left(\Delta_{a}^{*}\right)^{\dagger}\left(M_{-}^{2}\right)_{a b} \Delta_{b}^{*}$. 


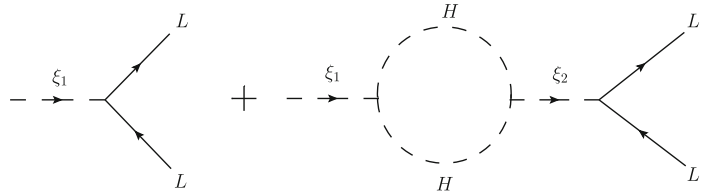

Fig. 3 The tree and one loop self energy diagrams in decay of $\xi_{1}$

The trilinear couplings $\mu \overline{\left(H^{c}\right)} i \tau^{2} \Delta^{\dagger} H+$ h.c. in Eq. 3 then becomes $\sum_{a=1,2} \mu_{a} \overline{\left(H^{c}\right)} i \tau^{2} \Delta^{\dagger} H+h . c$. .

The mass matrix in Eq. 23 can be given as:

$M_{ \pm}^{2}=\left(\begin{array}{cc}M_{1}^{2}-i C_{11} & -i C_{12}^{ \pm} \\ -i C_{21}^{ \pm} & M_{2}^{2}-i C_{22}\end{array}\right)$,

where $C_{a b}^{+}=\Gamma_{a b} M_{b}, C_{a b}^{-}=\Gamma_{a b}^{*} M_{b}$ and $C_{a a}=\Gamma_{a a} M_{a}$ with

$\Gamma_{a b} M_{b}=\frac{1}{8 \pi}\left(\mu_{1 a} \mu_{2 b}^{*}+M_{a} M_{b} \sum_{\alpha \beta} \lambda_{1 \alpha \beta} \lambda_{1 \alpha \beta}^{*}\right)$.

Diagonalizing the above mass matrix Eq. 24, we get two mass eigenvalues $M_{1}$ and $M_{2}$ corresponding to the two eigenstates $\xi_{1}^{ \pm}$and $\xi_{2}^{ \pm}$. Note that the mass eigenstates $\xi_{1}^{+}$and $\xi_{1}^{-}$ are not $\mathrm{CP}$ conjugate states of each other even though they are degenerate. Similarly $\xi_{2}^{+}$and $\xi_{2}^{-}$are not CP conjugate states, but they are degenerate. Therefore, the decay of these states can generate lepton asymmetry. We assume $M_{1} \ll M_{2}$. As a result the asymmetry created by $\xi_{2}^{ \pm}$decay will be erased by the lepton number violating process mediated by $\xi_{1}^{ \pm}$at the temperature below the mass of $\xi_{2}^{ \pm}$and finally we are left with only $\xi_{1}^{ \pm}$which generate the lepton asymmetry of the Universe.

The interference between tree and one loop self energy diagram of the process $\xi_{1}$ to $L L^{c}$ gives the $\mathrm{CP}$ asymmetry as shown in Fig. 3.

The asymmetry $\epsilon_{L}$ is estimated to be [6]

$$
\begin{aligned}
\epsilon_{L} & =\frac{\Gamma\left(\xi_{1} \rightarrow L L^{c}\right)-\Gamma\left(\xi_{1}^{c} \rightarrow L^{c} L\right)}{\Gamma_{1}} \\
& =\frac{\operatorname{Im}\left(\mu_{1} \mu_{2}^{*} \sum_{\alpha \beta} \lambda_{1 \alpha \beta} \lambda_{1 \alpha \beta}^{*}\right)}{8 \pi^{2}\left(M_{2}^{2}-M_{1}^{2}\right)}\left(\frac{M_{1}}{\Gamma_{1}}\right) .
\end{aligned}
$$

where we assume $M_{1} \ll M_{2}$. As a result, below the mass scale of $\xi_{1}$, we get a net $B-L$ asymmetry [121-124]:

$\left(n_{B-L}\right)_{\text {total }}=\epsilon_{L} \kappa s \times \frac{n_{\xi_{1}}^{e q}(T \rightarrow \infty)}{s}$,

where $\left(n_{\xi_{1}}^{e q} / s\right)(T \rightarrow \infty)=135 \zeta(3) /\left(4 \pi^{4} g_{*}\right)$ is the relativistic equilibrium abundance of $\xi_{1}$. The $\kappa$ is a washout factor, arises via inverse decay and scattering processes and $s=\left(2 \pi^{2} / 45\right) g_{*} T^{3}$ is the entropy density. Depending on the strength of Yukawa coupling, the value of $\kappa$ can vary between 0 to 1 . By solving the Boltzmann equations one can precisely calculate the value of $\kappa[6,7]$. The evolution of $X_{\xi_{1}}=n_{\xi_{1}} / s$ is given by the Boltzmann equation

$$
\frac{d X_{\xi_{1}}}{d z}=-\frac{\Gamma_{D}}{z H(z)}\left(X_{\xi_{1}}-X_{\xi_{1}}^{e q}\right)-\frac{\Gamma_{a}}{z H(z)}\left(\frac{X_{\xi_{1}}^{2}-X_{\xi_{1}}^{e q 2}}{X_{\xi_{1}}^{e q}}\right) .
$$

Here the temperature dependent decay rate is given by:

$$
\begin{aligned}
\Gamma_{D} & =\Gamma_{1} \frac{K_{1}(z)}{K_{2}(z)}, \text { where } \Gamma_{1} \\
& =\frac{1}{8 \pi} \frac{\left|m_{v}\right| M_{1}^{2}}{v^{2} \sqrt{B_{L} B_{H}}}, \text { and } H(z)=\frac{H\left(T=M_{1}\right)}{z^{2}} .
\end{aligned}
$$

The $B_{L, H}$ are the branching ratios of $\xi_{1} \rightarrow L L$ and $\xi_{1} \rightarrow$ $H H$ respectively such that $B_{L}+B_{H}=1$. The $K_{1,2}$ are the modified Bessel functions. In Eq. 28, $\Gamma_{a}=\gamma_{a} / n_{\xi_{1}}^{e q}$, where the $\gamma_{a}$ 's are the scattering densities for various processes and are given by:

$$
\begin{aligned}
& \gamma\left(\xi_{1}^{+} \xi_{1}^{-} \rightarrow \bar{f} f\right)=\frac{M_{1}^{4}\left(6 g^{4}+5 g^{\prime 4}\right)}{128 \pi^{5} z} \\
& \quad \times \int_{x_{\text {min }}}^{\infty} d x \sqrt{x} K_{1}(z \sqrt{x}) r^{3}, \\
& \gamma\left(\xi_{1}^{+} \xi_{1}^{-} \rightarrow H^{\dagger} H\right)=\frac{M_{1}^{4}\left(g^{4}+g^{\prime 4} / 2\right)}{512 \pi^{5} z} \\
& \quad \times \int_{x_{\min }}^{\infty} d x \sqrt{x} K_{1}(z \sqrt{x}) r^{3}, \\
& \gamma\left(\xi_{1}^{+} \xi_{1}^{-} \rightarrow W^{a} W^{b}\right)=\frac{M_{1}^{4} g^{4}}{64 \pi^{5} z} \int_{x_{\min }}^{\infty} d x \sqrt{x} K_{1}(z \sqrt{x}) \\
& \quad \times\left[r(5+34 / x)-\frac{24}{x^{2}}(x-1) \ln \left(\frac{1+r}{1-r}\right)\right], \\
& \gamma\left(\xi_{1}^{+} \xi_{1}^{-} \rightarrow B B\right)=\frac{3 M_{1}^{4} g^{\prime 4}}{128 \pi^{5} z} \int_{x_{\min }}^{\infty} d x \sqrt{x} K_{1}(z \sqrt{x}) \\
& \times\left[r(1+4 / x)-\frac{4}{x^{2}}(x-2) \ln \left(\frac{1+r}{1-r}\right)\right]
\end{aligned}
$$

where $z=M_{1} / T, r=\sqrt{1-4 / x}$ and $x=\hat{s} / M_{1}^{2}$ with $\hat{s}$ is the Mandelstam variable, the center of mass energy. In Eq. 30, $g, g^{\prime}$ are the gauge couplings corresponding to $S U(2)_{L}$ and $U(1)_{Y}$ respectively. The $n_{\xi_{1}}^{e q}=\frac{g_{d o f} M_{1}^{2} T}{2 \pi^{2}} K_{2}\left(M_{1} / T\right)$.

The abundance of $Y_{\xi_{1}}=\left(n_{\xi_{1}^{-}}-n_{\xi_{1}^{+}}\right) / s$, due to the decay and inverse decay of $\xi_{1}$ particles, satisfy the Boltzmann equation: 

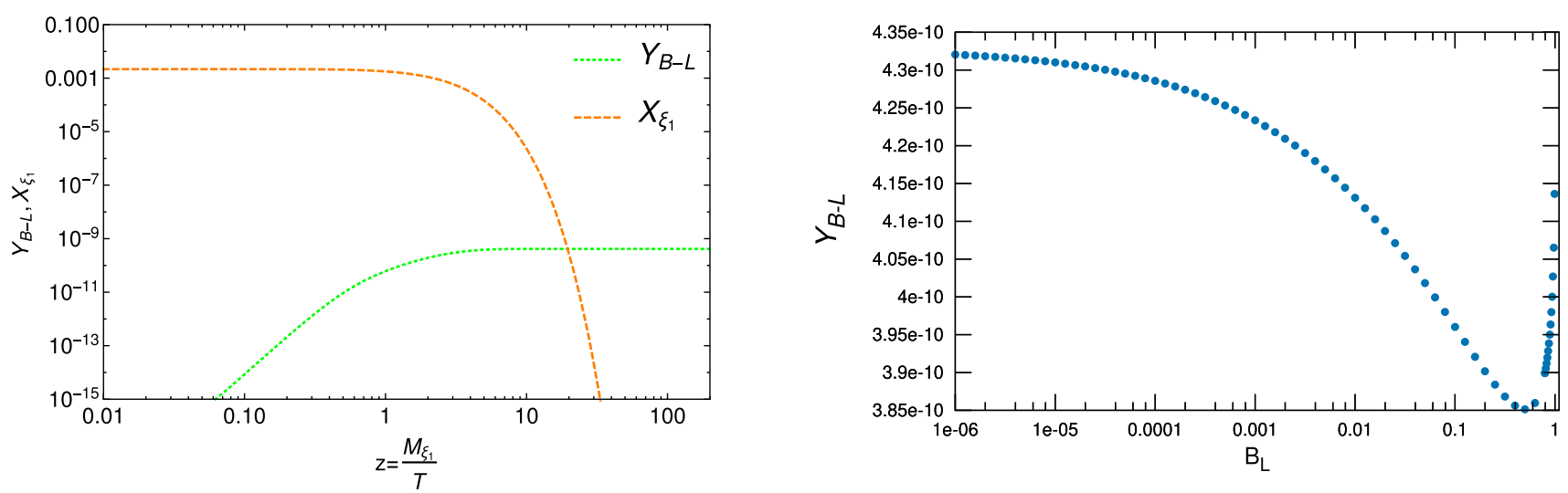

Fig. 4 Left). The abundance of $Y_{B-L}$ as a function of dimensionless variable $z=M_{1} / T$. We consider the parameter space $B_{L}=0.99, B_{H}=0.01$, $\epsilon_{L} \sim 10^{-6}$. Right). The dependence of branching ratio, $B_{L}$, on $Y_{B-L}$ abundance

$\frac{d Y_{\xi_{1}}}{d z}=-\frac{\Gamma_{D}}{z H(z)} Y_{\xi_{1}}+\sum_{i=B-L, H} \frac{\Gamma_{I D}^{i}}{z H(z)} B_{i} Y_{i}$

where

$\Gamma_{I D}^{i}=\Gamma_{D}\left(X_{\xi_{1}}^{e q} / X_{i}^{e q}\right)$ and $B_{i}=\Gamma_{i} / \Gamma_{1}$

Due to the conservation of hypercharge, the above Boltzmann equations satisfy the relation: $2 Y_{\xi}+\sum_{i} Y_{i}=0$ [6], where $i=B-L, H$. The evolution of $Y_{i}=n_{i} / s$, with $i=B-L, H$, is then given by,

$$
\begin{aligned}
\frac{d Y_{i}}{d z}= & 2\left\{\frac{\Gamma_{D}}{z H(z)}\left[\epsilon_{i}\left(X_{\xi_{1}}-X_{\xi_{1}}^{e q}\right)\right]+B_{i}\left(\frac{\Gamma_{D}}{z H(z)} Y_{\xi_{1}}\right.\right. \\
& \left.\left.-\frac{\Gamma_{I D}^{i}}{z H(z)} 2 Y_{i}\right)-\frac{\Gamma_{S}}{z H(z)} \frac{X_{\xi_{1}}^{e q}}{X_{L}^{e q}} 2 Y_{L}\right\} .
\end{aligned}
$$

The $\Gamma_{s}=\gamma_{s} / n_{\xi_{1}}^{e q}$ is the scattering rate of lepton number violating process, $L L \rightarrow H H$.

In the left panel of Fig. 4 we show the $B-L$ abundance $Y_{B-L}$ generated by the lightest triplet. We see that the $B-L$ yield agrees with the Planck observation [5]. In the right panel of Fig. 4 we show the contribution of branching ratio $B_{L}$ to the abundance of $Y_{B-L}$. As the $B_{L}$ increases (which implies increasing dilution factor) the $Y_{B-L}$ decreases gradually until $B_{L} \approx 0.5$. For $B_{L}>0.5, Y_{B-L}$ increases due to $\Gamma_{1} \propto$ $1 / \sqrt{B_{L} B_{H}}$ as given in Eq. 29 .

Above electroweak phase transition a part of the $B-L$ asymmetry gets transferred to the baryon (B) asymmetry via the $B+L$ violating sphaleron transitions. The remaining $B-L$ asymmetry gets transferred to the dark sector by a higher dimensional operator [93]:

$\mathcal{O}_{8}=\frac{1}{M_{\text {asy }}^{4}} \bar{\chi}^{2}(L H)^{2}$
Note that this operator conserves $B-L$ and remains in thermal equilibrium above electroweak phase transition. As a result, the operator $\mathcal{O}_{8}$, redistributes the $B-L$ asymmetry between visible (comprising of SM fields) and dark (comprising of $\chi$ ) sectors. Since the DM candidate $\chi$ carries a Lepton number, $L=1$, we find that the lowest dimension operator allowed by the symmetry of our model that can transfer asymmetry from visible sector to dark sector is $\mathcal{O}_{8}=\bar{\chi}^{2}(L H)^{2} / M_{\text {asy }}^{4}$. Other possible lower dimensional operators, for example, dim-7, dim- 6 and dim-5 allowed in this model, are not able to transfer the asymmetry from one sector to the other. So those operators will not alter the phenomenology of the asymmetric dark matter. See for instance Ref. [125].

The $B-L$ asymmetry of the visible sector gets transferred to a net baryon asymmetry via sphaleron transitions which conserve $B-L$ but violate $B+L$. The $B-L$ asymmetry of dark sector remains untouched and we see it as relics of DM of the present Universe. The symmetric component of the DM $\chi$ gets annihilated to SM fields through $\phi-H$ mixing which we study in the next section.

Note that the $B-L$ transfer operator $\mathcal{O}_{8}$ will decouple from the thermal plasma at different temperatures, depending on the value of $M_{a s y}$. The decoupling temperature can be found by comparing the interaction rate of the operator with the Hubble expansion parameter. At the decoupling temperature $T_{D}$, the interaction rate $\Gamma_{\mathrm{D}}$ of the transfer operator $\mathcal{O}_{8}$ can be given as,

$\Gamma_{\mathrm{D}} \simeq\left(\frac{T_{D}^{4}}{M_{\mathrm{asy}}^{4}}\right)^{2} T_{D}$.

By comparing $\Gamma_{\mathrm{D}}$ with the Hubble expansion parameter $H(T)$ at a temperature $\mathrm{T}_{\mathrm{D}}$ we get

$M_{\text {asy }}^{8}>M_{\mathrm{Pl}} T_{D}^{7}$ 
This condition also implies that the processes allowed by the transfer operator will remain out of equilibrium below electroweak phase transition. Note that when $\chi$ mass is much smaller than the decoupling temperature $T_{D}$, then only the estimation of Eq. 36 holds.

Through out the above calculation we assume that the mass scale of the triplet scalar should be high enough so that the lepton asymmetry, generated by it, can be converted to the baryon asymmetry via sphaleron transitions before the latter processes decouple from thermal bath. That is the mass scale of triplet scalar $M_{\Delta}$ should be greater than the sphaleron decoupling temperature $T_{\mathrm{sph}} \approx 80+0.45 M_{h_{1}}=136.33>$ $M_{W}$ [93,126-128]. This implies that $M_{\Delta} \gg M_{W}$. In our case, we assume $T_{D} \gtrsim T_{\text {sph }}$ i.e., the asymmetry transfer operator decouples before sphaleron processes decouple, which constrains $M_{\text {asy }}$ using Eq. 36 to be $M_{\text {asy }}>0.9 \times 10^{4} \mathrm{GeV}$.

We assume that, the DM $\chi$ is in thermal contact with the visible sector until the $T_{\text {sph }}>M_{W}$ through the higher dimensional operator $\mathcal{O}_{8}$. Therefore we get the number density of $\chi$ asymmetry (see Appendix A), which is nothing but the $B-L$ number density in dark sector, to be

$n_{\chi}=\left(n_{\mathrm{B}-\mathrm{L}}\right)_{\mathrm{dark}}=-2 \mu_{\chi}=\frac{58}{291}\left(n_{B-L}\right)_{\mathrm{vis}}$.

We also get the baryon asymmetry generated by the $\mathrm{CP}$ violating out-of-equilibrium decay of $\xi_{1}$ in the visible sector as

$n_{\mathrm{B}}=\frac{30}{97}\left(n_{\mathrm{B}-\mathrm{L}}\right)_{\mathrm{vis}}$

Therefore, the total $B-L$ number density of the Universe generated by the CP-violating out of equilibrium decay of the scalar triplet $\xi_{1}$, is the sum of $n_{B-L}$ in the visible and dark sectors and is given by

$$
\begin{aligned}
\left(n_{B-L}\right)_{\mathrm{total}} & =\left(n_{B-L}\right)_{\mathrm{vis}}+\left(n_{B-L}\right)_{\mathrm{dark}} \\
& =\frac{349}{291}\left(n_{B-L}\right)_{\mathrm{vis}} .
\end{aligned}
$$

Comparing Eq. 39 with Eq. 27 and using Eq. 38, we get the required $\mathrm{CP}$ asymmetry for observed lepton asymmetry $\epsilon_{L}=141.23(\eta / \kappa)\left(s / n_{\xi_{1}}^{e q}(T \rightarrow \infty)\right)$. Thus for $\kappa \sim 0.01$ we get $\epsilon_{L} \sim 10^{-6}$. Using Eq. 39 in Eqs. 38 and 37, we get,

$n_{\mathrm{B}}=\frac{90}{349}\left(n_{\mathrm{B}-\mathrm{L}}\right)_{\text {total }}, \quad n_{\chi}=\frac{58}{349}\left(n_{B-L}\right)_{\text {total }}$

The present day ratio of DM relic density to baryon relic density, given by WMAP and the PLANCK data $[4,5]$, is $\Omega_{\mathrm{DM}} h^{2} / \Omega_{\mathrm{B}} h^{2}=5.35 \pm 0.07$. This implies from Eq. 40 that,

$$
M_{\chi}=\frac{\Omega_{\mathrm{DM}} h^{2}}{\Omega_{\mathrm{B}} h^{2}}\left(n_{B} / n_{\chi}\right) M_{p} \approx 7.8 \mathrm{GeV}
$$

where $M_{p}$ is the proton mass and $n_{B} / n_{\chi}=90 / 58$. Here onwards we discuss the constraints on the annihilation crosssection $\sigma(\bar{\chi} \chi \rightarrow S M)$ which depletes the symmetric component of the $\chi$-DM, for $M_{\chi} \approx 7.8 \mathrm{GeV}$.

\subsection{Depletion of symmetric component of the DM}

The symmetric component of $\chi$ can be efficiently annihilated through the $\phi-H$ mixing portal to the SM particles. Due to Breit-Wigner enhancement in the cross-section, when extra scalar mass $\left(M_{h_{2}}\right)$ is twice of the DM mass, we get depletion of the symmetric component of the DM candidate. The annihilation cross-section of the process: $\bar{\chi} \chi \rightarrow \bar{f} f$, where $f$ is a SM fermion, is given by,

$$
\begin{aligned}
\sigma_{\chi}= & \frac{\sqrt{s-4 M_{f}^{2}}}{16 \pi s \sqrt{s}} \\
& \times \frac{\lambda_{D M}^{2} \lambda_{f}^{2} \cos ^{2} \gamma \sin ^{2} \gamma}{\left[\left(s-M_{h_{1}}^{2}\right)^{2}+\Gamma_{h_{1}}^{2} M_{h_{1}}^{2}\right]\left[\left(s-M_{h_{2}}^{2}\right)^{2}+\Gamma_{h_{2}}^{2} M_{h_{2}}^{2}\right]} \\
& \times\left\{\left[2 s-\left(M_{h_{1}}^{2}+M_{h_{2}}^{2}\right)\right]^{2}+\left[\Gamma_{h_{1}} M_{h_{1}}+\Gamma_{h_{2}} M_{h_{2}}\right]^{2}\right\} \\
& \times\left\{\left(s-2 M_{\chi}^{2}\right)\left(s-2 M_{f}^{2}\right)-2 M_{f}^{2}\left(s-2 M_{\chi}^{2}\right)\right. \\
& \left.-2 M_{\chi}^{2}\left(s-2 M_{f}^{2}\right)+4 M_{\chi}^{2} M_{f}^{2}\right\},
\end{aligned}
$$

where $\sqrt{s}$ is the center of mass energy and $\lambda_{f}=M_{f} / v$ with $M_{f}$ being the mass of SM fermion $f$. The decay width of $h_{1}$ is given by:

$\Gamma_{h_{1}}=\cos ^{2} \gamma \Gamma_{h_{1}}^{S M}+\sin ^{2} \gamma \Gamma_{h_{1}}^{\bar{\chi} \chi}+\Gamma_{h_{1}}^{h_{2} h_{2}}$,

where $\Gamma_{h_{1}}^{S M}=4.2 \mathrm{MeV}$ and for $\Gamma_{h_{1}}^{\bar{\chi} \chi}, h_{2} h_{2}, \Gamma_{h_{2}}$ refer to [124]. The thermal averaged annihilation cross-section of $\bar{\chi} \chi \rightarrow$ $\bar{f} f$ is then given by [129]:

$\left\langle\sigma_{\chi} v\right\rangle=\frac{1}{8 M_{\chi}^{4} T K_{2}^{2}\left(M_{\chi} / T\right)} \times \int_{4 M_{\chi}^{2}}^{\infty} \sigma_{\chi}\left(s-4 M_{\chi}^{2}\right) \sqrt{s} K_{1}(\sqrt{s} / T) d s$

where $K_{1}$ and $K_{2}$ are modified Bessel functions of first and second kind respectively and $\mathrm{T}$ is the temperature of thermal bath.

As we discussed at the end of previous Sect. $4, \bar{\chi} \chi$ annihilates dominantly to the pairs of $\bar{b} b, \bar{\tau} \tau$ and $\bar{c} c$ particles. The unknown parameters which dominantly contribute to the annihilation cross-section in Eq. 42 are the mass of $h_{2}$, i.e. $M_{h_{2}}$ and $\phi-H$ mixing, i.e. $\sin \gamma$ and the coupling of $h_{2}$ with $\chi$, i.e. $\lambda_{D M}$. All these parameters are constrained by invisible Higgs decay [130], relic abundance of DM measured by Planck [5] and WMAP [4], and spin-independent direct detection cross-section at XENON100 [131], LUX [132] 

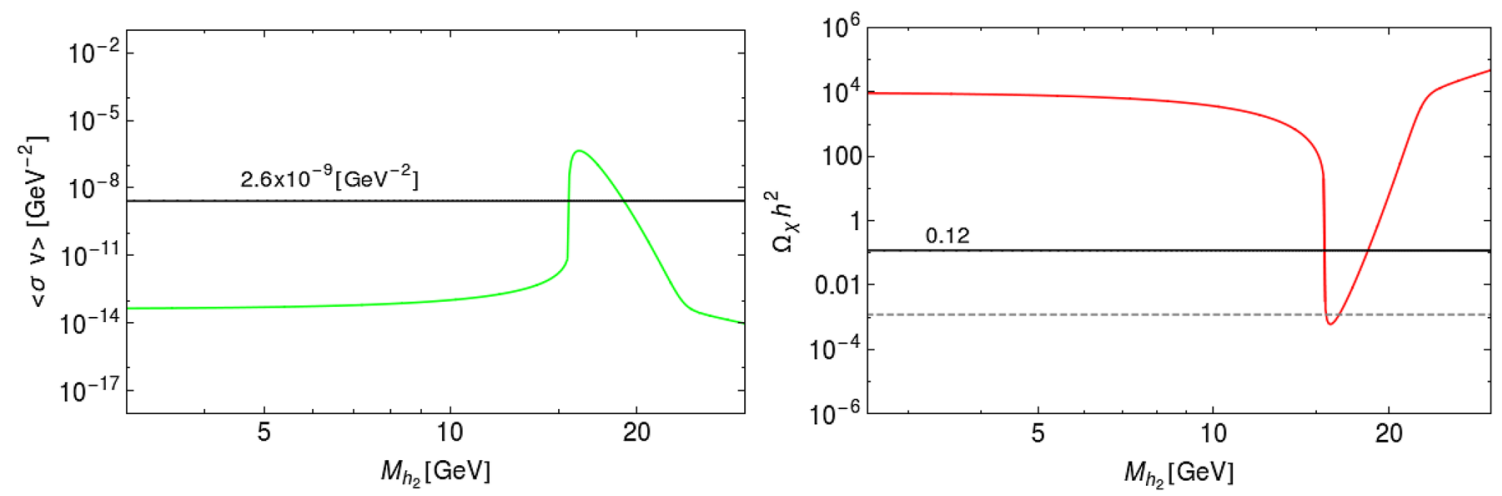

Fig. $5 \sigma v(\bar{\chi} \chi \rightarrow \bar{f} f)$ vs. $M_{h_{2}}$ (left plot) and the corresponding $\Omega_{\chi} h^{2}$ vs. $M_{h_{2}}$ (right plot) are shown for $\lambda_{\mathrm{DM}}=2 \times 10^{-2}$ and $\sin \gamma=0.16$. In the right plot, the horizontal Black solid line and Gray dashed line correspond to $\Omega_{\chi} h^{2}=0.12$ (i.e. $100 \%$ of DM relic) and $\Omega_{\chi} h^{2}=0.0012$ (i.e. $1 \%$ of DM relic) respectively

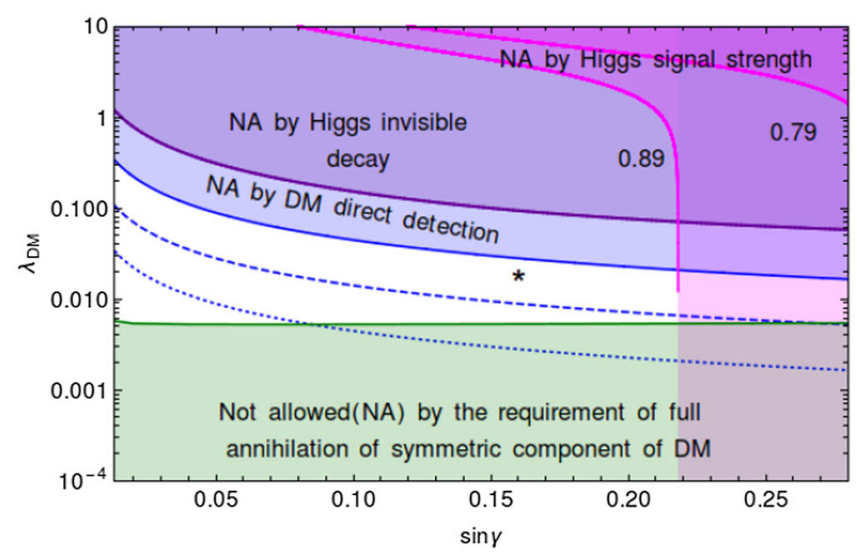

Fig. $6 \lambda_{D M}$ versus $\sin \gamma$ parameter space. The region above the Blue, Purple and Pink lines are disallowed by the spin independent dark matter nucleon cross-section of $10^{-43} \mathrm{~cm}^{2}$ at XENON1T [134] for DM mass $7.8 \mathrm{GeV}$, the invisible Higgs decay, i.e. $B r_{\text {inv }} \geq 24 \%$ and the Higgs signal strength for $\mu=0.79,0.89$, respectively. The region below the bottom green line is not allowed $\langle\sigma v\rangle<2.6 \times 10^{-9} / \mathrm{GeV}^{2}$ because

and XENON1T [133,134], and the measurement of signal strength of the Higgs particle at LHC $[135,136]$. As discussed in Sect. 2, we set $\sin \gamma=0.16$. Moreover, we fixed $\lambda_{D M}=2 \times 10^{-2}$ to estimate the value of cross-section $\left\langle\sigma_{\chi} v\right\rangle$. We then plotted the thermal averaged annihilation cross-section $\left\langle\sigma_{\chi} \mid v\right\rangle$ as a function of $M_{h_{2}}$ in Fig. 5. Here we fixed $M_{\chi} / T=20$ as we expect the maximum annihilation of $\bar{\chi} \chi \rightarrow \bar{f} f$ occurs at a temperature $T=M_{\chi} / 20$. From the left plot of Fig. 5, we see that in most of the parameter space $\left\langle\sigma_{\chi}|v|\right\rangle$ is less than the $\left\langle\sigma_{\chi}|v|\right\rangle_{F}=2.6 \times 10^{-9} / \mathrm{GeV}^{2}$, but near the resonance region, it satisfy the condition $\left\langle\sigma_{\chi}|v|\right\rangle>$ $\left\langle\sigma_{\chi}|v|\right\rangle_{F}$. Note that a large cross-section is required to completely annihilate the symmetric component of the DM and it can be achieved near the resonance, where the mass of $h_{2}$ is nearly twice of the DM mass. Far from the resonance we have $\left\langle\sigma_{\chi}|v|\right\rangle\left\langle\left\langle\sigma_{\chi}|v|\right\rangle_{F}\right.$. Therefore, we get an over abundance of DM. In the right plot of Fig. 5, we have shown relics

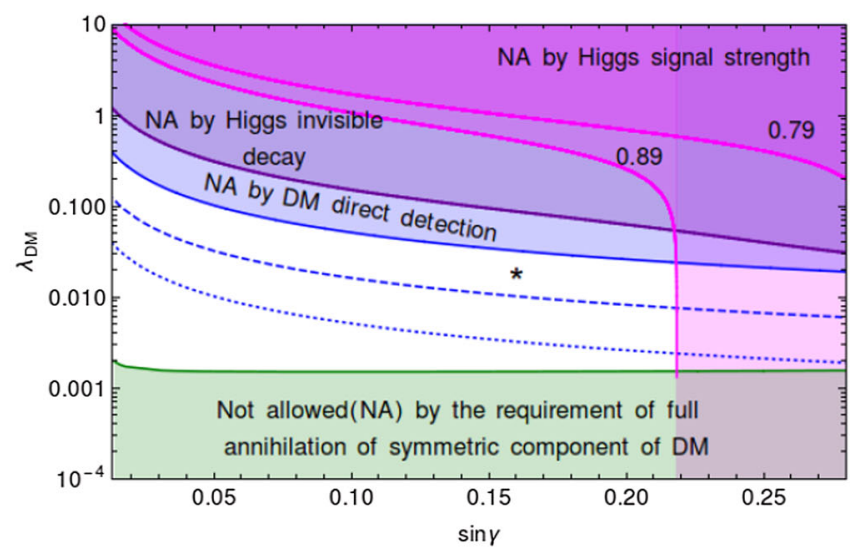

it does not satisfy the full annihilation of the symmetric component of the DM. The Left and Right panels correspond to $M_{h_{2}}=15.6 \mathrm{GeV}$ and $16.7 \mathrm{GeV}$ respectively. In both panels we fixed $M_{\chi}=7.8 \mathrm{GeV}$. The star corresponds to a point $\lambda_{D M}=2 \times 10^{-2}$ and $\sin \gamma=0.16$. The Blue dashed and dotted lines correspond to DM-nucleon cross-sections of $10^{-44} \mathrm{~cm}^{2}$ and $10^{-45} \mathrm{~cm}^{2}$ respectively for $M_{\chi}=7.8 \mathrm{GeV}$

of symmetric component of $\chi$ as a function of $M_{h_{2}}$. We see that the symmetric component of DM can be annihilated significantly only when $M_{h_{2}}$ varies in the range $15.6-16.7$ $\mathrm{GeV}$. This shows that our result crucially depends on $M_{h_{2}}$. We will come back to this point while giving a summary plot in Fig. 6.

\subsection{Higgs signal strength}

The Higgs signal strength is defined for a particular channel $h_{1} \rightarrow x x$ as

$$
\begin{aligned}
\mu_{h_{1} \rightarrow x x} & =\frac{\sigma_{h_{1}}}{\sigma_{h_{1}}^{\mathrm{SM}}} \frac{\operatorname{Br}_{h_{1} \rightarrow x x}}{\operatorname{Br}_{h_{1} \rightarrow x x}^{\mathrm{SM}}} \\
& =\frac{\cos ^{4} \gamma \Gamma_{h_{1}}^{S M}}{\Gamma_{h_{1}}},
\end{aligned}
$$


where $\Gamma_{h_{1}}$ is given in Eq. 43. The $\sigma_{h_{1}}$ and $\sigma_{h_{1}}^{\mathrm{SM}}$ are Higgs production cross section in BSM model and SM respectively. Higgs signal strength measurement at LHC can constrain $\phi-H$ mixing in our model. Currently the combined Higgs signal strength measured value is $\mu=1.17 \pm 0.1[135,136]$. We have taken $2 \sigma$ and $3 \sigma$ deviations from the best fit value to constrain our model parameters, the corresponding contour lines are shown in Fig. 6.

\subsection{Constraints from invisible Higgs decay}

This model allows SM-like Higgs $h_{1}$ to decay via invisible channels through $\phi-H$ mixing: $h_{1} \rightarrow h_{2} h_{2}, h_{1} \rightarrow \bar{\chi} \chi$. The branching ratio for the invisible Higgs decay can be defined as:

$B r_{\text {inv }}=\frac{\sin ^{2} \gamma \Gamma_{h_{1}}^{\bar{\chi} \chi}+\left[\operatorname{Br}\left(h_{2} \rightarrow \bar{\chi} \chi\right)\right] \Gamma_{h_{1}}^{h_{2} h_{2}}}{\cos ^{2} \gamma \Gamma_{h_{1}}^{S M}+\sin ^{2} \gamma \Gamma_{h_{1}}^{\bar{\chi} \chi}+\Gamma_{h_{1}}^{h_{2} h_{2}}}$

Note that LHC gives an upper bound to the invisible Higgs decay as $B r_{\text {inv }} \leq 24 \%$ [130]. This bound on Higgs invisible decay width constraint the $\lambda$ DM and $\sin \gamma$ in our model as shown in Fig. 6.

\subsection{Constraints from direct detection of DM}

In our setup, the $\phi-H$ scalar mixing allows the DM $\chi$ to scatter off the target nucleus which can be checked at terrestrial laboratories. The spin independent DM-nucleon elastic scattering cross-section per nucleon can be written as [137-143],

$\sigma^{S I}=\frac{\mu_{r}^{2}}{\pi A^{2}}\left[Z f_{p}+(A-Z) f_{n}\right]^{2}$

where the $\mathrm{A}$ and $\mathrm{Z}$ are the mass and atomic numbers of the target nucleus and $\mu_{r}$ is the reduced mass and is given by $\mu_{r}=M_{\chi} m_{N} /\left(M_{\chi}+m_{N}\right)$, where $m_{N}$ is the mass of the nucleon (proton or neutron). In Eq. $47 f_{p}$ and $f_{n}$ are the effective interaction strengths of DM with proton and neutron of the target nucleus and are given by:

$f_{p, n}=\sum_{q=u, d, s} f_{T_{q}}^{p, n} \alpha_{q} \frac{m_{p, n}}{m_{q}}+\frac{2}{27} f_{T G}^{p, n} \sum_{q=c, t, b} \alpha_{q} \frac{m_{p, n}}{m_{q}}$,

where

$\alpha_{q}=\lambda_{D M}\left(\frac{m_{q}}{v}\right)\left[\frac{1}{M_{h_{2}}^{2}}-\frac{1}{M_{h_{1}}^{2}}\right] \sin \gamma \cos \gamma$.

In Eq. 48, the $f_{T_{q}}^{p, n}$ are the quark mass fractions inside the nucleons, defined as $m_{N} f_{T_{q}}^{p, n} \equiv\left\langle N\left|m_{q} \bar{q} q\right| N\right\rangle$ and their values are $f_{T u}^{(p)}=0.020 \pm 0.004, f_{T d}^{(p)}=0.026 \pm 0.005, f_{T s}^{(p)}=$
$0.118 \pm 0.062, f_{T u}^{(n)}=0.014 \pm 0.003, f_{T d}^{(n)}=0.036 \pm$ $0.008, f(n)_{T s}=0.118 \pm 0.062[124,143]$. The coupling strength of DM with the gluons in the target nuclei is parameterized as

$f_{T G}^{p, n}=1-\sum_{q=u, d, s} f_{T_{q}}^{p, n}$.

In Fig. 6 we combined all the constraints coming from the invisible Higgs decay, Higgs signal strength, direct detection of DM at XENON1T [134] and the relic abundance of dark matter in the plane of $\lambda_{\mathrm{DM}}$ versus $\sin \gamma$. In the leftpanel of Fig. 6 we have taken $M_{h_{2}}=15.6 \mathrm{GeV}$, while that in the right panel $M_{h_{2}}=16.7 \mathrm{GeV}$. The Pink shaded region shows the constraint from Higgs signal strength measurement ( $\mu=1.17 \pm 0.1$ ) [135,136], the Purple region shows the constraint from invisible Higgs decay (i.e., $B r_{\text {inv }} \geq 24 \%$ ) [130], while the Blue region is disallowed by the spin independent direct detection cross-section at XENON1T where we have used DM-nucleon cross-section $10^{-43} \mathrm{~cm}^{2}$ for a DM mass $7.8 \mathrm{GeV}$, and the Green region is disallowed by the constraint coming from the relic abundance of DM (i.e., $\left.\langle\sigma v(\bar{\chi} \chi \rightarrow \bar{f} f)\rangle<2.6 \times 10^{-9} / \mathrm{GeV}^{2}\right)$. Finally, we end up with a white region of allowed parameter space in the plane of $\lambda_{\text {DM }}$ versus $\sin \gamma$. In the white region, the projected Blue dashed and dotted lines, which correspond to a DM-nucleon cross-section of $10^{-44} \mathrm{~cm}^{2}$ and $10^{-45} \mathrm{~cm}^{2}$ respectively for a DM mass $M_{\chi}=7.8 \mathrm{GeV}$, can be probed by future data.

\section{Signature of light Higgs}

In our model, apart from the SM Higgs boson $h_{1}$, there exist an extra scalar particle $h_{2}$, which plays an important role in the annihilation of symmetric component of the dark matter. As discussed before, the mass of $h_{2}$ is required to be around $16 \mathrm{GeV}$ to annihilate the symmetric component of the dark matter. Here we briefly discuss the collider signature of a light Higgs pertinent to this model.

At LHC, the main production channel of $h_{2}$ is through the decay of SM Higgs $h_{1}$, i.e. $h_{1} \rightarrow h_{2} h_{2}$. The branching fraction of $h_{1} \rightarrow h_{2} h_{2}$ is about $3.58 \%$ (for a typical set of values: $\sin \gamma=0.16, \lambda_{\mathrm{DM}}=0.01, \lambda_{H}=0.129, \lambda_{\mathrm{H} \phi}=0.01$, $M_{h_{2}}=16.42 \mathrm{GeV}$ ). The subsequent decay of $h_{2}$ to SM particles can be studied at collider. We note that the main decay modes of $h_{2}$ in this model are $\operatorname{Br}\left(h_{2} \rightarrow b \bar{b}\right) \sim 74.15 \%$, $\operatorname{Br}\left(\mathrm{h}_{2} \rightarrow \tau^{+} \tau^{-}\right) \sim 6.51 \%, B r\left(h_{2} \rightarrow c \bar{c}\right) \sim 10.43 \%$, $\operatorname{Br}\left(h_{2} \rightarrow \mu^{+} \mu^{-}\right) \sim 0.0741 \%$ and $\operatorname{Br}\left(h_{2} \rightarrow \bar{\chi} \bar{\chi}\right) \sim$ $8.90 \%$. At LHC the main production channel of the SM Higgs is gluon-fusion and the corresponding cross-section for $g g \rightarrow h_{1} \rightarrow h_{2} h_{2}$ is given by $1.78 \mathrm{pb}$ [144] at c.m.energy $14 \mathrm{TeV}$. Depending on the decay mode the final state will be vary, such as $b \bar{b} b \bar{b}, b \bar{b} \tau^{+} \tau^{-}, \tau^{+} \tau^{-} \tau^{+} \tau^{-}, \tau^{+} \tau^{-} \mu^{+} \mu^{-}$, etc., 
for the search of $h_{2}$ at LHC. All of these channels have either large backgrounds, which are mostly dominated by the QCD, or very small cross-section. So at LHC, it is very difficult to search this light Higgs boson. Despite this a large number of searches have been performed in the last years. A most recent search result is given in [145]]. For $M_{h_{2}}=16 \mathrm{GeV}$ the bound on $\operatorname{Br}\left(h_{1} \rightarrow h_{2} h_{2} \rightarrow b \bar{b} \mu^{+} \mu^{-}\right) \lesssim 10^{-4}$, which is compatible with the branching ratios mentioned above. There are other existing searches by CMS and ATLAS for various final states in the different mass range of $h_{2}$. For example, $4 \mu$ in the final state with $M_{h_{2}}$ varying in the range 1-15 $\mathrm{GeV}$ [146,147], $2 \mu 2 \tau$ in the final state with $M_{h_{2}}$ varying in the range 3.6-21 GeV [148], two muons and two tracks in the final state with $M_{h_{2}}$ varying in the range 4-15 GeV [149], two bottom quarks and two tau leptons in the final state with $M_{h_{2}}$ varying in the range 15-60 GeV [150], four bottoms in the final state with $M_{h_{2}}$ varying in the range $15-30 \mathrm{GeV}$ [151], $4 \gamma$ in the final state with $M_{h_{2}}$ varying in the range 10-62 $\mathrm{GeV}[152], \gamma \gamma j j$ in the final state with $M_{h_{2}}$ varying in the range: $20-60 \mathrm{GeV}$ [153], etc. However, none of the searches are fruitful yet.

The signature of the light scalar $h_{2}$ can also be studied with a better precession at leptonic colliders, such as the International Linear Collider (ILC), which is proposed to run at the center of mass energies $500 \mathrm{GeV}$ and $1 \mathrm{TeV}$. The main production channel of the light Higgs $h_{2}$ at ILC is via the process $e^{+} e^{-} \rightarrow Z h_{1}$ and subsquesnt decays of $h_{1} \rightarrow h_{2} h_{2}$ [154]. Alternatively the direct production of $h_{2}$ can happen through the process: $e^{+} e^{-} \rightarrow Z h_{2}[154,155]$. Subsequently these particles decay to SM particles and pave a way to detect $h_{2}$. In fact, the analysis of Ref. [155] shows that ILC can even be able to detect a $10 \mathrm{GeV} h_{2}$ with a $\phi-H$ mixing of order $10^{-2}$

\section{Conclusion}

In this paper, we studied a simultaneous explanation of visible and dark matter in a type-II seesaw scenario, thus explaining why DM to baryon ratio in the present Universe is about a factor of five. We extended the standard model with two triplet scalars $\Delta_{i},(i=1,2)$ and a singlet leptonic Dirac fermion $\chi$. The particle $\chi$ is charged under an extended global symmetry $U(1)_{D}$, which is softly broken by dimension-8 operator $(\bar{\chi} L H)^{2} / M_{\text {asy }}^{4}$ to a remnant $Z_{2}$ symmetry under which $\chi$ is odd, while all other particles are even. As a result $\chi$ served as a stable DM candidate.

The lightest triplet scalar creates a net $B-L$ asymmetry in the early Universe via its CP violating out-of-equilibrium decay to SM leptons and Higgs. The created $B-L$ asymmetry is then transferred to the dark sector via the dimension- 8 operator, which conserves $B-L$ number. The transfer of $B-L$ asymmetry is active until the dimension- 8 operator decouples from the thermal bath. As a result, there is a proportionality arises between the net $B-L$ asymmetry in the visible and the dark sector. Note that the dimension- 8 operator decouples from the thermal bath before the decoupling of sphaleron processes. As a result, the $B-L$ asymmetry in the visible sector gets converted to a net B-asymmetry through the sphaleron transitions, while the $B-L$ asymmetry of dark sector remains untouched which we see today as relics of asymmetric $\chi$-particles. A singlet scalar $\phi$ is then introduced to deplete the symmetric component of the DM $\chi$ through its mixing with the SM-Higgs. We found that nearly $100 \%$ depletion of the symmetric component of $\chi$-DM is possible within a narrow range of singlet scalar mass, namely (15.616.7) $\mathrm{GeV}$, where DM mass is about half of singlet scalar mass, i.e., $M_{\chi} \sim 8 \mathrm{GeV}$. By considering the constraints from invisible Higgs decay, Higgs signal strength, null detection of DM at XENON1T and relic abundance of DM, we showed the allowed region of parameters in the plane of DM coupling $\lambda_{D M}$ and $H-\phi$ scalar mixing: $\sin \gamma$.

After electroweak phase transition, the scalar triplet $\Delta$ acquires an induced vacuum expectation value $\langle\Delta\rangle$. As a result the sub-eV neutrino masses could be explained through the $\Delta L L$ coupling, assuming $\mu \sim M_{\Delta} \sim 10^{14} \mathrm{GeV}$.

Acknowledgements The computations was supported in part by the SAMKHYA: High Performance Computing Facility provided by Institute of Physics, Bhubaneswar.

Data Availability Statement This manuscript has no associated data or the data will not be deposited. [Authors' comment: All the data generated during this study are contained in this published article.]

Open Access This article is licensed under a Creative Commons Attribution 4.0 International License, which permits use, sharing, adaptation, distribution and reproduction in any medium or format, as long as you give appropriate credit to the original author(s) and the source, provide a link to the Creative Commons licence, and indicate if changes were made. The images or other third party material in this article are included in the article's Creative Commons licence, unless indicated otherwise in a credit line to the material. If material is not included in the article's Creative Commons licence and your intended use is not permitted by statutory regulation or exceeds the permitted use, you will need to obtain permission directly from the copyright holder. To view a copy of this licence, visit http://creativecomm ons.org/licenses/by/4.0/.

Funded by SCOAP ${ }^{3}$.

\section{Appendix A: Asymmetry transfer from visible to dark sector}

The asymmetry in the equilibrium number densities of particle $n_{i}$ over antiparticle $\bar{n}_{i}$ can be written as

$$
n_{i}-\bar{n}_{i}=\frac{g_{i}}{2 \pi^{2}} \int_{0}^{\infty} d q q^{2}\left[\frac{1}{e^{\frac{E_{i}(q)-\mu_{i}}{T}} \pm 1}-\frac{1}{e^{\frac{E_{i}(q)+\mu_{i}}{T}} \pm 1}\right],
$$


where the $g_{i}$ is the internal degrees of freedom of the particle species $i$. In the above equation $E_{i}$ and $q_{i}$ represent the energy and momentum of the particle species $i$. In the approximation of a weakly interacting plasma, where $\beta \mu_{i} \ll 1, \beta \equiv 1 / T$ (for further detailed discussion visit [93],[89]) we get

$$
\begin{aligned}
n_{i}-\bar{n}_{i} & \sim \frac{g_{i} T^{3}}{6} \times\left[2 \beta \mu_{i}+\mathcal{O}\left(\left(\beta \mu_{i}\right)^{3}\right) \quad\right. \text { for bosons } \\
& \sim \frac{g_{i} T^{3}}{6} \times\left[\beta \mu_{i}+\mathcal{O}\left(\left(\beta \mu_{i}\right)^{3}\right) \quad\right. \text { for fermions }
\end{aligned}
$$

In our model, the asymmetry transfer operator is given by $\mathcal{O}_{8}=\frac{1}{M_{\text {asy }}^{4}} \bar{\chi}^{2}(L H)^{2}$. Depending on the value of the $M_{\text {asy }}$ the operator will decouple from thermal plasma at different temperatures. Since the $B-L$ asymmetry generated by the decay of scalar triplet is required to be transferred to the dark sector via $\mathcal{O}_{8}$ operator, we assume the decoupling temperature $T_{D}$ of the latter to be $T_{t}>T_{D}>T_{W}$, where $T_{t}$ is the temperature of thermal bath when the top quark decouples and $T_{W}$ is the temperature when the $W$ boson decouples from the thermal plasma. In this case the effective Lagrangian for Yukawa coupling is given by:

$\mathcal{L}_{Y u k a w a}=g_{e_{i}}^{k} \bar{e}_{i L} h^{k} e_{i R}+g_{u_{i}}^{k} \bar{u}_{i L} h^{k} u_{i R}+g_{d_{i}}^{k} \bar{d}_{i L} h^{k} d_{i R}+h . c$.

where $k=1,2$ for two Higgses $h_{1}$ and $h_{2}$. As Higgs field is real so the above Lagrangian gives the following chemical equilibrium condition:

$0=\mu_{h}=\mu_{u_{L}}-\mu_{u_{R}}=\mu_{d_{L}}-\mu_{d_{R}}=\mu_{e_{L}}-\mu_{e_{R}}$

The charged current interaction part of the SM Lagrangian after electroweak symmetry breaking is given by:

$\mathcal{L}_{i n t}^{(W)}=g W_{\mu}^{+} \bar{u}_{L} \gamma^{\mu} d_{L}+g W_{\mu}^{+} e_{L} \gamma^{\mu} \bar{v}_{e L}$.

The above equation implies that the charged current interactions remain in thermal equilibrium until W-boson decouples from thermal bath. As a result we get the following chemical potential constraints:

$\mu_{W}=\mu_{u_{L}}-\mu_{d_{L}}$

and

$\mu_{W}=\mu_{v}-\mu_{e_{L}}$.

The electroweak sphalerons remain in thermal equilibrium until a temperature $T_{\mathrm{sph}} \gtrsim T_{W}$. As a result we get a constraint:

$\mu_{u_{L}}+2 \mu_{d_{L}}+\mu_{v}=0$.
At a temperature below electroweak phase transition, the electric charge neutrality of the Universe holds. However, at the epoch: $T_{t}>T_{D}>T_{W}$, the top quark is already decoupled from the thermal plasma and hence does not take part in the charge neutrality condition. Therefore, we get

$Q=4\left(\mu_{u_{L}}+\mu_{u_{R}}\right)+6 \mu_{W}-3\left(\mu_{d_{L}}+\mu_{d_{R}}+\mu_{e_{L}}+\mu_{e_{R}}\right)=0$.

Now using the Eqs. 54-59, the baryon and lepton number density $n_{B}$ and $n_{L}$ can be written as,

$n_{B}=-\frac{90}{19} \mu_{v}$

and

$n_{L}=\frac{201}{19} \mu_{\nu}$

where we have dropped the common factor $g T^{3} \beta / 6$ as we are interested in ratio of densities, rather than their individual values. The net $B-L$ asymmetry in the visible sector is thus given by:

$\left(n_{B-L}\right)_{\mathrm{vis}}=-\frac{291}{19} \mu_{v}$

After sphaleron processes decouple at $T_{\mathrm{sph}}$, the baryon and lepton number densities would be conserved separately. As a result Eqs. 54-62 would remain valid at $T_{\text {sph }}>T_{D}>M_{W}$. Once the sphaleron processes decouple, the ratio of $n_{B} / n_{B-L}$ would be frozen. As a result from Eqs. 60 and 62, it can be written as,

$$
\begin{aligned}
\frac{n_{B_{\text {final }}}}{\left(n_{B-L}\right)_{\mathrm{vis}}} & =\frac{n_{B}}{\left(n_{B-L}\right)_{\mathrm{vis}}}=\frac{30}{97}=0.31 \\
n_{B_{\text {final }}} & =0.31\left(n_{B-L}\right)_{\mathrm{vis}} .
\end{aligned}
$$

As the asymmetry transfer operator $\mathcal{O}_{8}$ is active down to W-boson decoupling temperature, a part of the $B-L$ asymmetry gets transferred from visible sector to the dark sector. Therefore, $\left(n_{B-L}\right)_{\text {vis }}$ is no longer equal to the $\left(n_{B-L}\right)_{\text {total }}$.

The equilibration of $\mathcal{O}_{8}$ operator gives the constraint:

$\mu_{\chi}=\mu_{v}$.

As a result the number density of dark matter $\chi$, which is nothing but the $B-L$ number density of dark sector, is given by:

$n_{\chi}=-2 \mu_{\chi}=\frac{58}{291}\left(n_{B-L}\right)_{\mathrm{vis}}=\left(n_{B-L}\right)_{\mathrm{dark}}$

We use the $B-L$ number density of $\chi$ to calculate its mass in Sect. 4. 


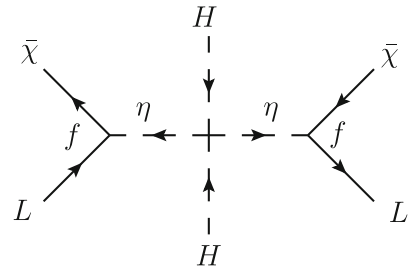

Fig. 7 Feynman diagram of the dimension- 8 operator

\section{Appendix B: A viable origin of dimension-8 operator}

We now discuss a viable origin of the dimension- 8 operator, $\mathcal{O}_{8}=\frac{\bar{\chi}^{2}(L H)^{2}}{M_{\text {asy }}^{4}}$, which conserves $B-L$ symmetry but breaks $U(1)_{D}$ global symmetry explicitly to a remnant $Z_{2}$ symmetry under which the DM $\chi$ is odd. Apart from the singlet fermion $\chi$, we add a relatively heavy scalar doublet (under $\left.S U(2)_{L}\right) \eta$ to the dark sector. We assume that $\eta$ is odd under $Z_{2}$ symmetry and possesses same charge under $U(1)_{D}$ as that of $\chi$. As a result the relevant Lagrangian, which can give rise to the required dimension- 8 operator, can be given as:

$\mathcal{L} \supset f \bar{\chi} L \eta+\lambda_{\eta H}\left(\eta^{\dagger} H\right)^{2}+$ h.c.,

where $\eta=\left(\eta_{1}+i \eta_{2}\right) / \sqrt{2}$.

The Feynman diagram, which in the effective theory can give rise to a dimension-8 operator, is shown in Fig. 7. We assume that $\eta$ is heavy and doesn't acquire a vev in order to preserve the remnant $Z_{2}$ symmetry of the dark sector. As a result, integrating $\eta$, we get the required dimension- 8 operator:

$\frac{f^{2} \lambda_{\eta H} \bar{\chi} L H H \bar{\chi} L}{M_{\eta}^{4}} \equiv \frac{\bar{\chi}^{2}(L H)^{2}}{M_{\text {asy }}^{4}}$,

where $M_{\text {asy }}^{4}=M_{\eta}^{4} /\left(f^{2} \lambda_{\eta H}\right)$.

With the introduction of $\eta$ the additional terms in potential are

$M_{\eta}^{2}\left(\eta^{\dagger} \eta\right)+\lambda_{\eta}\left(\eta^{\dagger} \eta\right)^{2}+\lambda_{\eta H}\left(\eta^{\dagger} \eta\right)\left(H^{\dagger} H\right)+\lambda_{\eta H}\left(\eta^{\dagger} H\right)^{2}+$ h.c. .

The last term $\lambda_{\eta H}\left(\eta^{\dagger} H\right)^{2}+$ h.c. in the potential breaks the $U(1)_{D}$ symmetry. This term will generate a mass splitting between $\eta_{1}$ and $\eta_{2}$. The breaking is related to the mass splitting and does not have any impact on other part of the model.

\section{References}

1. G. Jungman, M. Kamionkowski, K. Griest, Phys. Rep. 267, 195373 (1996). arXiv:hep-ph/9506380
2. G. Bertone, D. Hooper, J. Silk, Phys. Rep. 405, 279-390 (2005). arXiv:hep-ph/0404175

3. N. Aghanim et al., [Planck], Astron. Astrophys. 641, A1 (2020). arXiv:1807.06205 [astro-ph.CO]

4. G. Hinshaw et al., [WMAP], Astrophys. J. Suppl. 208, 19 (2013). arXiv: 1212.5226 [astro-ph.CO]

5. N. Aghanim et al., [Planck], Astron. Astrophys. 641, A6 (2020). arXiv:1807.06209 [astro-ph.CO]

6. C. Arina, N. Sahu, Nucl. Phys. B 854, 666-699 (2012). arXiv:1108.3967 [hep-ph]

7. C. Arina, J.O. Gong, N. Sahu, Nucl. Phys. B 865, 430-460 (2012). arXiv:1206.0009 [hep-ph]

8. C. Arina, R.N. Mohapatra, N. Sahu, Phys. Lett. B 720, 130-136 (2013). arXiv:1211.0435 [hep-ph]

9. S. Nussinov, Phys. Lett. B 165, 55-58 (1985)

10. K. Griest, D. Seckel, Nucl. Phys. B 283, 681-705 (1987) [Erratum: Nucl. Phys. B 296, 1034-1036 (1988)]

11. R.S. Chivukula, T.P. Walker, Nucl. Phys. B 329, 445-463 (1990)

12. S. Dodelson, B.R. Greene, L.M. Widrow, Nucl. Phys. B 372, 467493 (1992)

13. S.M. Barr, Phys. Rev. D 44, 3062-3066 (1991)

14. D.B. Kaplan, Phys. Rev. Lett. 68, 741-743 (1992)

15. H.K. Dreiner, G.G. Ross, Nucl. Phys. B 410, 188-216 (1993). arXiv:hep-ph/9207221

16. T. Inui, T. Ichihara, Y. Mimura, N. Sakai, Phys. Lett. B 325, 392 400 (1994). arXiv:hep-ph/9310268

17. S.D. Thomas, Phys. Lett. B 356, 256-263 (1995). arXiv:hep-ph/9506274

18. N. Cosme, L. Lopez Honorez, M.H.G. Tytgat, Phys. Rev. D 72, 043505 (2005). arXiv:hep-ph/0506320

19. M.H.G. Tytgat, arXiv:hep-ph/0606140

20. R. Kitano, I. Low, Phys. Rev. D 71, 023510 (2005). arXiv:hep-ph/0411133

21. K. Agashe, G. Servant, JCAP 02, 002 (2005). arXiv:hep-ph/0411254

22. G.R. Farrar, G. Zaharijas, Phys. Rev. Lett. 96, 041302 (2006). arXiv:hep-ph/0510079

23. R. Kitano, H. Murayama, M. Ratz, Phys. Lett. B 669, 145-149 (2008). arXiv:0807.4313 [hep-ph]

24. E. Nardi, F. Sannino, A. Strumia, JCAP 01, 043 (2009). arXiv:0811.4153 [hep-ph]

25. H. An, S.L. Chen, R.N. Mohapatra, Y. Zhang, JHEP 03, 124 (2010). arXiv:0911.4463 [hep-ph]

26. T. Cohen, K.M. Zurek, Phys. Rev. Lett. 104, 101301 (2010). arXiv:0909.2035 [hep-ph]

27. J. Shelton, K.M. Zurek, Phys. Rev. D 82, 123512 (2010). arXiv:1008.1997 [hep-ph]

28. H. Davoudiasl, D.E. Morrissey, K. Sigurdson, S. Tulin, Phys. Rev. Lett. 105, 211304 (2010). arXiv:1008.2399 [hep-ph]

29. N. Haba, S. Matsumoto, Prog. Theor. Phys. 125, 1311-1316 (2011). arXiv:1008.2487 [hep-ph]

30. M.R. Buckley, L. Randall, JHEP 09, 009 (2011). arXiv: 1009.0270 [hep-ph]

31. P.H. Gu, M. Lindner, U. Sarkar, X. Zhang, Phys. Rev. D 83, 055008 (2011). arXiv:1009.2690 [hep-ph]

32. M. Blennow, B. Dasgupta, E. Fernandez-Martinez, N. Rius, JHEP 03, 014 (2011). arXiv:1009.3159 [hep-ph]

33. J. McDonald, Phys. Rev. D 83, 083509 (2011). arXiv:1009.3227 [hep-ph]

34. L.J. Hall, J. March-Russell, S.M. West, arXiv:1010.0245 [hep-ph]

35. J.J. Heckman, S.J. Rey, JHEP 06, 120 (2011). arXiv:1102.5346 [hep-th]

36. M.T. Frandsen, S. Sarkar, K. Schmidt-Hoberg, Phys. Rev. D 84, 051703 (2011). arXiv:1103.4350 [hep-ph]

37. S. Tulin, H.B. Yu, K.M. Zurek, JCAP 05, 013 (2012). arXiv:1202.0283 [hep-ph] 
38. K. Kohri, A. Mazumdar, N. Sahu, Phys. Rev. D 80, 103504 (2009). arXiv:0905.1625 [hep-ph]

39. K. Kohri, A. Mazumdar, N. Sahu, P. Stephens, Phys. Rev. D 80 , 061302 (2009). arXiv:0907.0622 [hep-ph]

40. K. Kohri, N. Sahu, Phys. Rev. D 88, 103001 (2013). arXiv:1306.5629 [hep-ph]

41. M.L. Graesser, I.M. Shoemaker, L. Vecchi, JHEP 10, 110 (2011). arXiv:1103.2771 [hep-ph]

42. D. Hooper, J. March-Russell, S.M. West, Phys. Lett. B 605, 228 236 (2005). arXiv:hep-ph/0410114

43. H. Iminniyaz, M. Drees, X. Chen, JCAP 07, 003 (2011). arXiv: 1104.5548 [hep-ph]

44. N. Haba, S. Matsumoto, R. Sato, Phys. Rev. D 84, 055016 (2011). arXiv: 1101.5679 [hep-ph]

45. Z. Kang, J. Li, T. Li, T. Liu, J.M. Yang, Eur. Phys. J. C 76(5), 270 (2016). arXiv:1102.5644 [hep-ph]

46. K. Blum, A. Efrati, Y. Grossman, Y. Nir, A. Riotto, Phys. Rev. Lett. 109, 051302 (2012). arXiv:1201.2699 [hep-ph]

47. M. Fujii, T. Yanagida, Phys. Lett. B 542, 80-88 (2002). arXiv:hep-ph/0206066

48. T. Banks, S. Echols, J.L. Jones, JHEP 11, 046 (2006). arXiv:hep-ph/0608104

49. T.R. Dulaney, P. FileviezPerez, M.B. Wise, Phys. Rev. D 83, 023520 (2011). arXiv:1005.0617 [hep-ph]

50. T. Cohen, D.J. Phalen, A. Pierce, K.M. Zurek, Phys. Rev. D 82, 056001 (2010). arXiv: 1005.1655 [hep-ph]

51. B. Dutta, J. Kumar, Phys. Lett. B 699, 364-367 (2011). arXiv:1012.1341 [hep-ph]

52. A. Falkowski, J.T. Ruderman, T. Volansky, JHEP 05, 106 (2011). arXiv: 1101.4936 [hep-ph]

53. J. March-Russell, M. McCullough, JCAP 03, 019 (2012). arXiv:1106.4319 [hep-ph]

54. M.L. Graesser, I.M. Shoemaker, L. Vecchi, arXiv:1107.2666 [hep-ph]

55. K. Kamada, M. Yamaguchi, Phys. Rev. D 85, 103530 (2012). arXiv:1201.2636 [hep-ph]

56. D.G.E. Walker, arXiv:1202.2348 [hep-ph]

57. B. Feldstein, A.L. Fitzpatrick, JCAP 09, 005 (2010). arXiv:1003.5662 [hep-ph]

58. J. March-Russell, J. Unwin, S.M. West, JHEP 08, 029 (2012). arXiv:1203.4854 [hep-ph]

59. Y. Cai, M.A. Luty, D.E. Kaplan, arXiv:0909.5499 [hep-ph]

60. H. An, S.L. Chen, R.N. Mohapatra, S. Nussinov, Y. Zhang, Phys. Rev. D 82, 023533 (2010). arXiv: 1004.3296 [hep-ph]

61. C. Kouvaris, P. Tinyakov, Phys. Rev. Lett. 107, 091301 (2011). arXiv:1104.0382 [astro-ph.CO]

62. M.R. Buckley, Phys. Rev. D 84, 043510 (2011). arXiv:1104.1429 [hep-ph]

63. S. Chang, L. Goodenough, Phys. Rev. D 84, 023524 (2011). arXiv:1105.3976 [hep-ph]

64. S. Profumo, L. Ubaldi, JCAP 08, 020 (2011). arXiv:1106.4568 [hep-ph]

65. H. Davoudiasl, D.E. Morrissey, K. Sigurdson, S. Tulin, Phys. Rev. D 84, 096008 (2011). arXiv: 1106.4320 [hep-ph]

66. I. Masina, F. Sannino, JCAP 09, 021 (2011). arXiv:1106.3353 [hep-ph]

67. T. Lin, H.B. Yu, K.M. Zurek, Phys. Rev. D 85, 063503 (2012). arXiv:1111.0293 [hep-ph]

68. M.R. Buckley, S. Profumo, Phys. Rev. Lett. 108, 011301 (2012). arXiv:1109.2164 [hep-ph]

69. H. Davoudiasl, R.N. Mohapatra, New J. Phys. 14, 095011 (2012). arXiv:1203.1247 [hep-ph]

70. N. Okada, O. Seto, Phys. Rev. D 86, 063525 (2012). arXiv:1205.2844 [hep-ph]

71. T. Hugle, M. Platscher, K. Schmitz, Phys. Rev. D 98(2), 023020 (2018). arXiv:1804.09660 [hep-ph]
72. K. Petraki, R.R. Volkas, Int. J. Mod. Phys. A 28, 1330028 (2013). arXiv:1305.4939 [hep-ph]

73. N. Narendra, N. Sahoo, N. Sahu, Nucl. Phys. B 936, 76-90 (2018). arXiv:1712.02960 [hep-ph]

74. K.M. Zurek, Phys. Rep. 537, 91-121 (2014). arXiv:1308.0338 [hep-ph]

75. N.F. Bell, K. Petraki, I.M. Shoemaker, R.R. Volkas, Phys. Rev. D 84, 123505 (2011). arXiv:1105.3730 [hep-ph]

76. K. Petraki, M. Trodden, R.R. Volkas, JCAP 02, 044 (2012). arXiv:1111.4786 [hep-ph]

77. B. von Harling, K. Petraki, R.R. Volkas, JCAP 05, 021 (2012). arXiv:1201.2200 [hep-ph]

78. S.J. Lonsdale, R.R. Volkas, Phys. Rev. D 97(10), 103510 (2018) arXiv: 1801.05561 [hep-ph]

79. E.A. Novikov, Mod. Phys. Lett. A 31(15), 1650092 (2016)

80. E.A. Novikov, Electron. J. Theor. Phys. 13(35), 79-90 (2016)

81. P. Agrawal, M. Mitra, S. Niyogi, S. Shil, M. Spannowsky, Phys. Rev. D 98(1), 015024 (2018). arXiv:1803.00677 [hep-ph]

82. E. Ma, U. Sarkar, Phys. Rev. Lett. 80, 5716-5719 (1998). arXiv:hep-ph/9802445

83. T. Hambye, E. Ma, U. Sarkar, Nucl. Phys. B 602, 23-38 (2001). arXiv:hep-ph/0011192

84. A.G. Akeroyd, M. Aoki, H. Sugiyama, Phys. Rev. D 77, 075010 (2008). arXiv:0712.4019 [hep-ph]

85. D. Aristizabal Sierra, M. Dhen, T. Hambye, JCAP 08, 003 (2014). arXiv: 1401.4347 [hep-ph]

86. A.D. Sakharov, Sov. Phys. Usp. 34(5), 392-393 (1991)

87. M.C. Chen, arXiv:hep-ph/0703087

88. C.S. Fong, E. Nardi, A. Riotto, Adv. High Energy Phys. 2012, 158303 (2012). arXiv:1301.3062 [hep-ph]

89. E.W. Kolb, M.S. Turner, Front. Phys. 69, 1-547 (1990)

90. M. Fukugita, T. Yanagida, Phys. Lett. B 174, 45-47 (1986)

91. M. Ibe, S. Matsumoto, T.T. Yanagida, Phys. Lett. B 708, 112-118 (2012). arXiv:1110.5452 [hep-ph]

92. D.E. Kaplan, M.A. Luty, K.M. Zurek, Phys. Rev. D 79, 115016 (2009). arXiv:0901.4117 [hep-ph]

93. W.Z. Feng, P. Nath, G. Peim, Phys. Rev. D 85, 115016 (2012). arXiv:1204.5752 [hep-ph]

94. W.Z. Feng, A. Mazumdar, P. Nath, Phys. Rev. D 88(3), 036014 (2013). arXiv:1302.0012 [hep-ph]

95. E. Aprile et al., [XENON1T], Phys. Rev. Lett. 121, 111302 (2018). arXiv: 1805.12562

96. G. Angloher et al., [CRESST], Eur. Phys. J. C 76(1), 25 (2016). arXiv:1509.01515 [astro-ph.CO]

97. Q. Arnaud et al., [EDELWEISS], Phys. Rev. D 97(2), 022003 (2018). arXiv:1707.04308 [physics.ins-det]

98. R. Agnese et al., [CDMS], Phys. Rev. Lett. 111(25), 251301 (2013). arXiv:1304.4279 [hep-ex]

99. C.E. Aalseth et al., CoGeNT, Phys. Rev. D 88, 012002 (2013). arXiv:1208.5737 [astro-ph.CO]

100. J. Barreto et al., DAMIC, Phys. Lett. B 711, 264-269 (2012). arXiv:1105.5191 [astro-ph.IM]

101. R. Agnese et al., [SuperCDMS], Phys. Rev. Lett. 112(24), 241302 (2014). arXiv:1402.7137 [hep-ex]

102. R. Agnese et al. [SuperCDMS], Phys. Rev. Lett. 116(7), 071301 (2016). arXiv:1509.02448 [astro-ph.CO]

103. A. Tan et al., [PandaX-II], Phys. Rev. Lett. 117(12), 121303 (2016). arXiv:1607.07400 [hep-ex]

104. D.Y. Akimov, H.M. Araujo, E.J. Barnes, V.A. Belov, A. Bewick, A.A. Burenkov, V. Chepel, A. Currie, L. DeViveiros, B. Edwards et al., Phys. Lett. B 709, 14-20 (2012). arXiv:1110.4769 [astroph.CO]

105. P. Agnes et al., DarkSide, Phys. Lett. B 743, 456-466 (2015). arXiv: 1410.0653 [astro-ph.CO]

106. V. Khachatryan et al., CMS, JHEP 10, 076 (2017). arXiv:1701.02032 [hep-ex] 
107. V. Khachatryan et al., CMS, Phys. Lett. B 752, 146 (2016). arXiv: 1506.00424

108. S. Chatrchyan et al., CMS, JHEP 07, 98 (2011). arXiv: 1106.2375

109. A.M. Sirunyan et al., [CMS], Phys. Lett. B 796, 131 (2019). arXiv: 1812.00380

110. G. Aad et al., [ATLAS], Eur. Phys. J. C 76(4), 210 (2016). arXiv:1509.05051 [hep-ex]

111. M. Aaboud et al., ATLAS, JHEP 06, 166 (2018). arXiv: 1802.03388

112. K. Kannike, Eur. Phys. J. C 72, 2093 (2012). arXiv:1205.3781

113. A. Arhrib, R. Benbrik, M. Chabab, G. Moultaka, M. Peyranere, L. Rahili et al., Phys. Rev. D 84, 095005 (2011). arXiv:1105.1925

114. W. Konetschny, W. Kummer, Phys. Lett. B 70, 433-435 (1977)

115. T.P. Cheng, L.F. Li, Phys. Rev. D 22, 2860 (1980)

116. G. Lazarides, Q. Shafi, C. Wetterich, Nucl. Phys. B 181, 287-300 (1981)

117. J. Schechter, J.W.F. Valle, Phys. Rev. D 22, 2227 (1980)

118. R.N. Mohapatra, G. Senjanovic, Phys. Rev. D 23, 165 (1981)

119. P. Fileviez Perez, T. Han, G.Y. Huang, T. Li, K. Wang, Phys. Rev. D 78, 015018 (2008). arXiv:0805.3536 [hep-ph]

120. Y. Cai, T. Han, T. Li, R. Ruiz, Front. Phys. 6, 40 (2018). arXiv:1711.02180 [hep-ph]

121. W. Buchmuller, M. Plumacher, Phys. Rep. 320, 329-339 (1999). arXiv:hep-ph/9904310

122. W. Buchmuller, P. Di Bari, M. Plumacher, Ann. Phys. 315, 305351 (2005). arXiv:hep-ph/0401240

123. G.F. Giudice, A. Notari, M. Raidal, A. Riotto, A. Strumia, Nucl. Phys. B 685, 89-149 (2004). arXiv:hep-ph/0310123

124. N. Narendra, S. Patra, N. Sahu, S. Shil, Phys. Rev. D 98(9), 095016 (2018). arXiv:1805.04860 [hep-ph]

125. D.E. Kaplan, M.A. Luty, K.M. Zurek, Phys. Rev. D 79, 115016 (2009)

126. D. Aristizabal Sierra, J.F. Kamenik, M. Nemevsek, JHEP 10, 036 (2010). arXiv: 1007.1907

127. A. Strumia, Nucl. Phys. B 809, 308 (2009). arXiv:0806.1630

128. Y. Burnier, M. Laine, M. Shaposhnikov, JCAP 02, 007 (2006). arXiv:hep-ph/0511246

129. P. Gondolo, G. Gelmini, Nucl. Phys. B 360, 145-179 (1991)

130. V. Khachatryan et al., CMS, JHEP 02, 135 (2017). arXiv:1610.09218 [hep-ex]

131. E. Aprile et al., XENON100, Phys. Rev. Lett. 109, 181301 (2012). arXiv:1207.5988 [astro-ph.CO]

132. D.S. Akerib et al., [LUX], Phys. Rev. Lett. 118(2), 021303 (2017). arXiv:1608.07648 [astro-ph.CO]

133. E. Aprile et al., [XENON], JCAP 04, 027 (2016). arXiv: 1512.07501 [physics.ins-det]

134. E. Aprile et al., XENON Collaboration, Phys. Rev. Lett 121, 111302 (2018). arXiv:1805.12562 [astro-ph.CO]
135. A.M. Sirunyan et al., Euro Phys. J. C 79(5), 421 (2019). arXiv: 1809.10733 (hep-ex)

136. G. Aad et al., ATLAS and CMS, JHEP 08, 045 (2016). arXiv:1606.02266 [hep-ex]

137. M.W. Goodman, E. Witten, Phys. Rev. D 31, 3059 (1985)

138. J.R. Ellis, K.A. Olive, C. Savage, Phys. Rev. D 77, 065026 (2008). arXiv:0801.3656 [hep-ph]

139. Y. Akrami, C. Savage, P. Scott, J. Conrad, J. Edsjo, JCAP 04, 012 (2011). arXiv:1011.4318 [astro-ph.CO]

140. S. Bhattacharya, N. Sahoo, N. Sahu, Phys. Rev. D 93(11), 115040 (2016). arXiv:1510.02760 [hep-ph]

141. S. Patra, S. Rao, N. Sahoo, N. Sahu, Nucl. Phys. B 917, 317-336 (2017). arXiv:1607.04046 [hep-ph]

142. S. Bhattacharya, N. Sahoo, N. Sahu, Phys. Rev. D 96(3), 035010 (2017). arXiv:1704.03417 [hep-ph]

143. J.R. Ellis, A. Ferstl, K.A. Olive, Phys. Lett. B 481, 304-314 (2000). arXiv:hep-ph/0001005

144. S. Dittmaier et al., [LHC Higgs Cross Section Working Group], arXiv:1101.0593 [hep-ph]

145. G. Add et al., arXiv: 2110.00313 (hep-ex)

146. M. Aaboud et al., [ATLAS], JHEP 06, 166 (2018). https://doi.org/ 10.1007/JHEP06(2018)166. arXiv:1802.03388 [hep-ex]

147. A.M. Sirunyan et al., [CMS], Phys. Lett. B 796, 131154 (2019). https://doi.org/10.1016/j.physletb.2019.07.013. arXiv: 1812.00380 [hep-ex]

148. A.M. Sirunyan et al., [CMS], JHEP 08, 139 (2020). https://doi. org/10.1007/JHEP08(2020)139. arXiv:2005.08694 [hep-ex]

149. A.M. Sirunyan et al., [CMS], Phys. Lett. B 800, 135087 (2020). https://doi.org/10.1016/j.physletb.2019.135087. arXiv:1907.07235 [hep-ex]

150. A.M. Sirunyan et al., [CMS], Phys. Lett. B 785, 462 (2018). https://doi.org/10.1016/j.physletb.2018.08.057 arXiv:1805.10191 [hep-ex]

151. G. Aad et al., [ATLAS], Phys. Rev. D 102(11), 112006 (2020). https://doi.org/10.1103/PhysRevD.102.112006. arXiv:2005.12236 [hep-ex]

152. G. Aad et al., [ATLAS], Eur. Phys. J. C 76(4), 210 (2016). https:// doi.org/10.1140/epjc/s10052-016-4034-8. arXiv:1509.05051 [hep-ex]

153. M. Aaboud et al., [ATLAS], Phys. Lett. B 782, 750767 (2018). https://doi.org/10.1016/j.physletb.2018.06.011. arXiv:1803.11145 [hep-ex]

154. P. Drechsel, G. Moortgat-Pick, G. Weiglein, Eur. Phys. J. C 80(10), 922 (2020). https://doi.org/10.1140/epjc/s10052-020-08438-1. arXiv:1801.09662 [hep-ph]

155. Y. Wang, M. Berggren, J. List, arXiv:2005.06265 [hep-ex] 